\title{
GEOCHEMICAL- MINERALOGICAL AND METALLOGENETICAL ASPECTS CONCERNING THE ORIGIN OF SEDIMENTS FROM LEG. 22 D.S.D.P DRILLED SITES 212 AND 213 IN EASTERN INDIAN OCEAN
}

\author{
Papavassiliou C. T. ${ }^{1}$ \\ ${ }^{I}$ National and Kapodistrian University of Athens, Faculty of Geology and Geoenvironment, \\ Department of Economic Geology and Geochemistry, papavas@geol.uoa.gr
}

\begin{abstract}
Basic mineralogical and geochemical data, are presented for sediment core samples from Deep Sea Drilling Project(D.S.D.P) boreholes :Sites 212 and 213 respectively, from the eastern Indian Ocean. In both Sites the Fe-Mn oxides are abundant controlling a numerous suite of trace elements. In Site 212 the Fe-Mn oxides are more abundant in the upper part of the borehole having mainly a diagenetic or hydrogenous origin, whilst in Site 213 these oxides are more abundant in the lower part having mainly a hydrothermal origin. In both Sites clay minerals are consisting mostly of the expandable mixed layered smectite/illite. The geochemical data shows that in both Sites the majority of the sediments have the characteristics of typical deep sea clay being both of detrital and authigenic origin. In Site 212 these clays with considerable amount of palygorskite and the zeolite clinoptilolite, both of authigenic origin, opaline silica, biogenous $\mathrm{CaCO}_{3}$, the $\mathrm{Fe}-\mathrm{Mn}$ oxides and some other detrital minerals together control the bulk chemical composition of the sediments. In Site 213 the clays with large amounts of $\mathrm{Fe}-\mathrm{Mn}$ oxides and considerable amounts of the zeolite Phillipsite and Palygorskite in small amounts and some other detrital minerals, control the chemical composition of the sediments. The increase in the Ti/Al and $\mathrm{Mg} / \mathrm{Al}$ ratios with depth in both Sites implies a volcanoclastic input in the bottom sediments probably from the underlying basaltic basement.
\end{abstract}

Key words: Deep sea sediments and clays, Fe-Mn oxides, authigenic minerals.

\section{Пврі́іпчи}

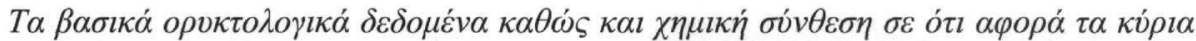

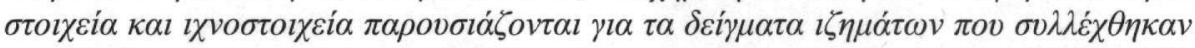

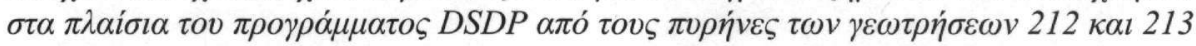

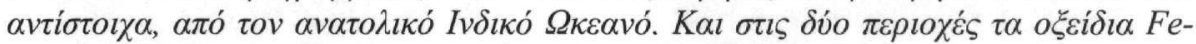

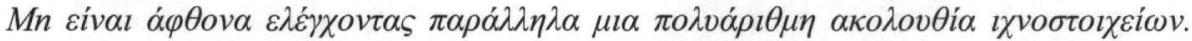

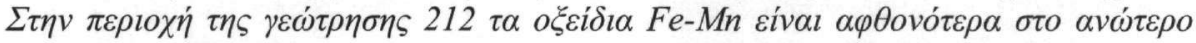

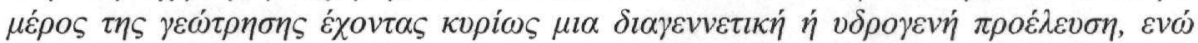

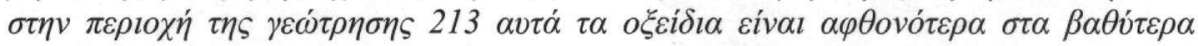

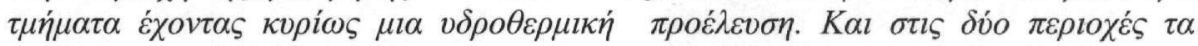

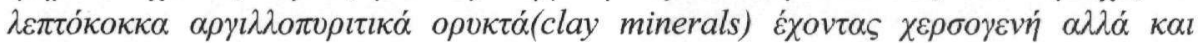




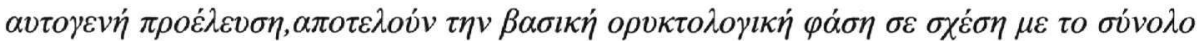

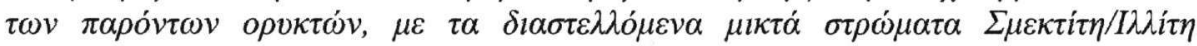

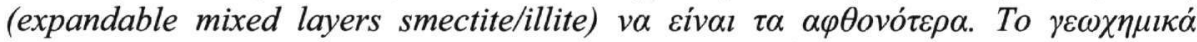

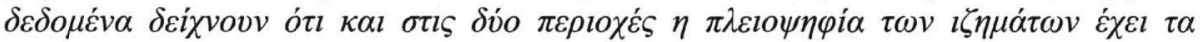

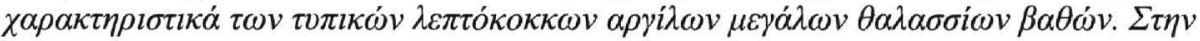

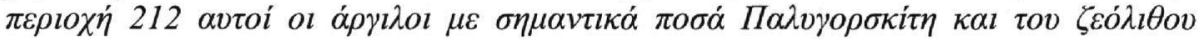

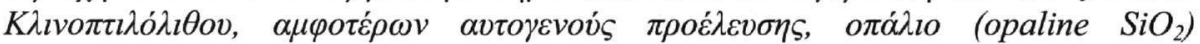

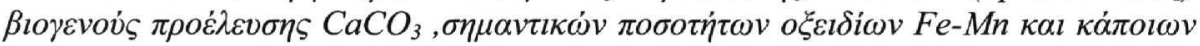

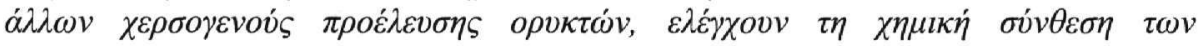

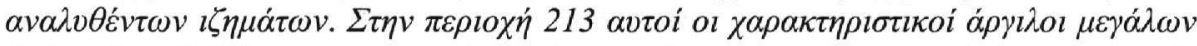

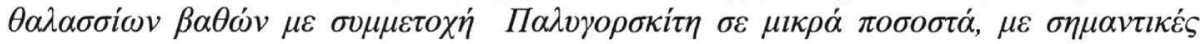

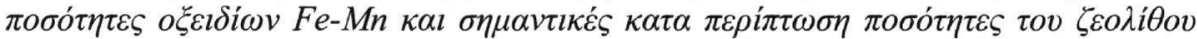

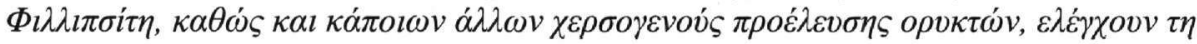

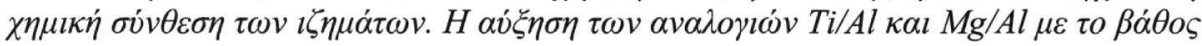

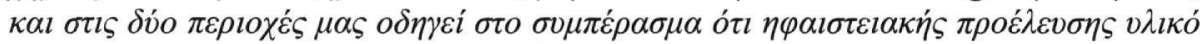

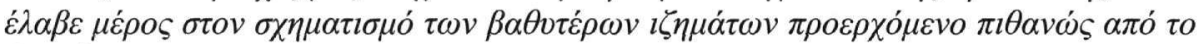

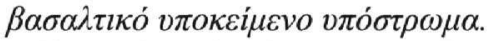

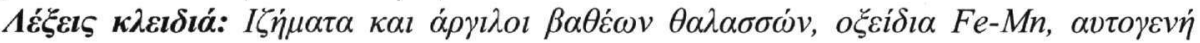

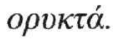

\section{Introduction}

During Leg.22 of the Deep-Sea Drilling Project (D.S.D.P) in Indian Ocean Sites 212 (latitude:9.7755 longitude:99.2973) and 213 (latitude -10.2118 longitude 93.8962) were drilled in a water depth of $6243 \mathrm{~m}$. and $5611 \mathrm{~m}$. respectively. Site 212 was drilled over the Wharton Basin, whilst Site 213 was drilled over the east site of Ninety-east Ridge of the Indian Ocean (Fig.1).

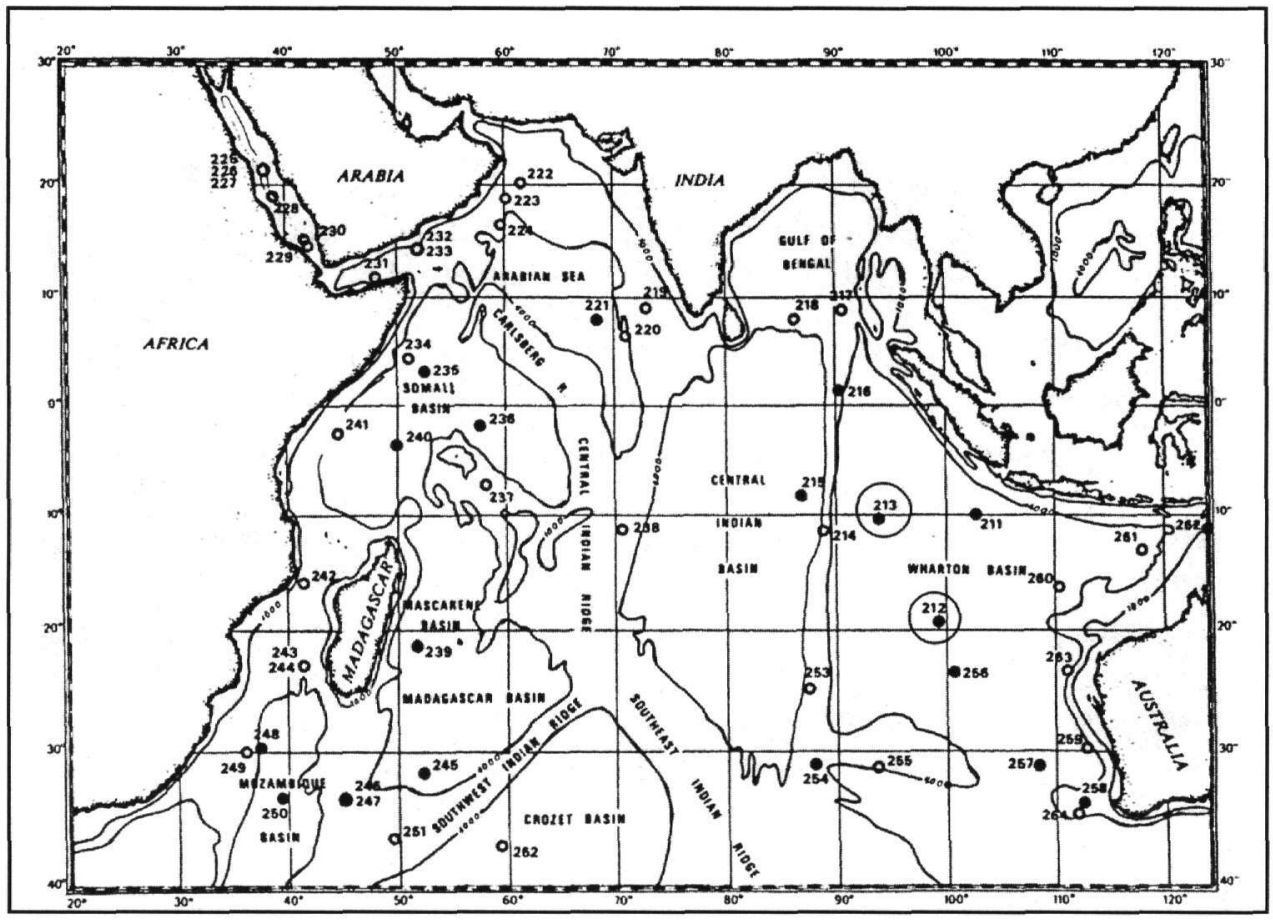

Figure 1 - D.S.D.P Glomar Challenger drill sites in Indian Ocean. The present study Sites 212 and 213 are shown by open circles 
The sedimentary sequence for both sites are summarised in Table 1 .

Table 1 - Generalised data of the main sedimentological characteristics of Sites 212 and 213 (based to Site reports Von Der Borch and Sclater 1974)

\begin{tabular}{|c|c|c|c|}
\hline SAMPLES & DEPTH BELOW SEA FLOOR(m.) & AGE & LITHOLOGY \\
\hline $212 / 15 / 2$ & 290,00 & M.MIOCENE & Fe-oxide rich BROWN ZEOLITIC CLAYSTONE \\
\hline $212 / 15 / 3$ & 292,00 & "4 & \\
\hline $212 / 16 / 1$ & 298,50 & " & \\
\hline $212 / 16 / 2$ & 300,00 & " & \\
\hline $212 / 17 \mathrm{ce}$ & 309,00 & 4 & \\
\hline $212 / 18 / 1$ & 317,50 & L.M.EOCENE & \\
\hline $212 / 18 / 2$ & 319,00 & "4 & MODERATE BROWN ZEOLITIC CLAYSTONE \\
\hline $212 / 23 / 5$ & 371,00 & LATE MIDDLE EOCENE & \\
\hline $212 / 27 / 1$ & 403,00 & $\overline{7}$ & NANNO CHALK-BROWN ZEOLITIC CLAYSTONE \\
\hline $212 / 28 / 2$ & 413,80 & $\overline{~ " ~}$ & Mn-Fe oxides rich BROWN ZEOLITIC CLAYSTONE \\
\hline $212 / 35 \mathrm{ec}$ & 485,50 & 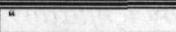 & FE OXIDE RICH CLAYSTONE \\
\hline $212 / 36 \mathrm{ec}$ & 489,00 & $\overline{~ " 4}$ & ZEOLITIC CLAYSTONE \\
\hline $212 / 37 / 1$ & 497,80 & $\overline{~ " 4}$ & BROWN ZEOLITIC CLAYSTONE \\
\hline $212 / 37 \mathrm{ec}$ & 498,50 & $\overline{~ " 4}$ & \\
\hline $212 / 38 / 1$ & 507,50 & " & \\
\hline $213 / 8 / 5$ & 73,00 & QUATERNARY & RAD-DIATOM OOZE WITH SOME CLAY \\
\hline $213 / 9 / 4$ & 81,00 & UPPER MIOCENE & ZEOLITIC RAD.BEARING CLAY \\
\hline $21399 \mathrm{cc}$ & 84,50 & LATE MIOCENE & Mn oxide rich RAD.BEARING ZEOLITIC CLAY \\
\hline $213 / 10 / 3$ & 89,50 & MIDDLEMIOCENE & Mn oxide rich RAD. BEARING ZEOLITIC CLAY \\
\hline $213 / 11 / 2$ & 97,00 & 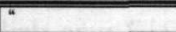 & Mn oxide rich ZEOLITIC CLAY \\
\hline $213 / 11 / 5$ & 10150 & " & Mn oxide rich ZEOLITIC CLAY \\
\hline $213 / 12 / 5$ & 110,80 & " & Mn-Fe oxides rich(goethite?) ZEOLITIC CLAY \\
\hline $213 / 13 / 2$ & 116,40 & " & Mn-Fe oxides rich ZEOLITIC CLAY \\
\hline $213 / 13 / 5$ & 120,50 & " & Mn-Fe oxides rich ZEOLITIC CLAY \\
\hline $213 / 4 / 3$ & 126,80 & E.EOCENE & RAD.RICH Mn-Fe oxides rich ZEOLITIC CLAY \\
\hline $213 / 15 / 2$ & 135,40 & E.EOCENE & NANNO BEARING Fe-oxides rich ZEOLITIC CLAY \\
\hline
\end{tabular}

Fifteen samples from the borehole cores of Site 212 and eleven samples from the borehole cores of Site 213 were chemically analysed for nine major elements and seventeen trace elements by an X-Ray Fluoresence Spectometer (Philips PW 1400); $\mathrm{CO}_{2}$ and $\mathrm{H}_{2} \mathrm{O}$ (expressed as loss of weight) were determined gravimetrically.

\section{Methods}

Chemical analyses of the samples, reduced to rock powder pellets, were produced by X-Ray Fluorescence Spetrometer (Philips PW 1400) at the School of Ocean and Earth Science of the University of Southampton. Water (expressed as loss of weight in the chemical analyses) and $\mathrm{CO}_{2}$ was determined gravimetrically.

All samples were visually inspected to verify the presence of biogenic phases (calcite and opaline silica). The chemical analyses were processed to correct for such phases, using calcite values and biogenic silica determined both semi-quantitavely.

Calcite was calculated according to a method proposed by Weijden et.al (2006) from total Ca using a correction for clay-bound $\left.\mathrm{Ca}: \mathrm{CaCO}_{3 \text { (sample) }}=2.5 \times \mathrm{Ca}_{\text {(sample) }}-(\mathrm{Ca} / \mathrm{Al})_{\text {clay }} \times \mathrm{Al}_{\text {sample }}\right)$ where $(\mathrm{Ca} / \mathrm{A} 1)$ clay= 0,345 (Turekian and Wedepohl, 1961); also the average of ratios in upper continental crust (Taylor and Mclennan, 1985) and North American shale (Gromet et al. 1984).

Biogenic silica values were determined according to a method proposed by Bostrom et al. (1976) which is based on the assumption that in sediments with little or no opaline silica the ratio $\mathrm{SiO}_{2} / \mathrm{Al}_{2} \mathrm{O}_{3}$ usually ranges between 3 and 4,the ratio found in average continental crust (Turekian 
and Wedepohl 1961); that is, the inorganic $\mathrm{Si} \approx 3 \mathrm{x}$ Al. The calculated data for both $\mathrm{CaCO}_{3}$ and opaline silica are listed in Table 2.

Table 2 - $\mathrm{CaCO}_{3}$ and Opaline Silica values determined semi-quantitatively

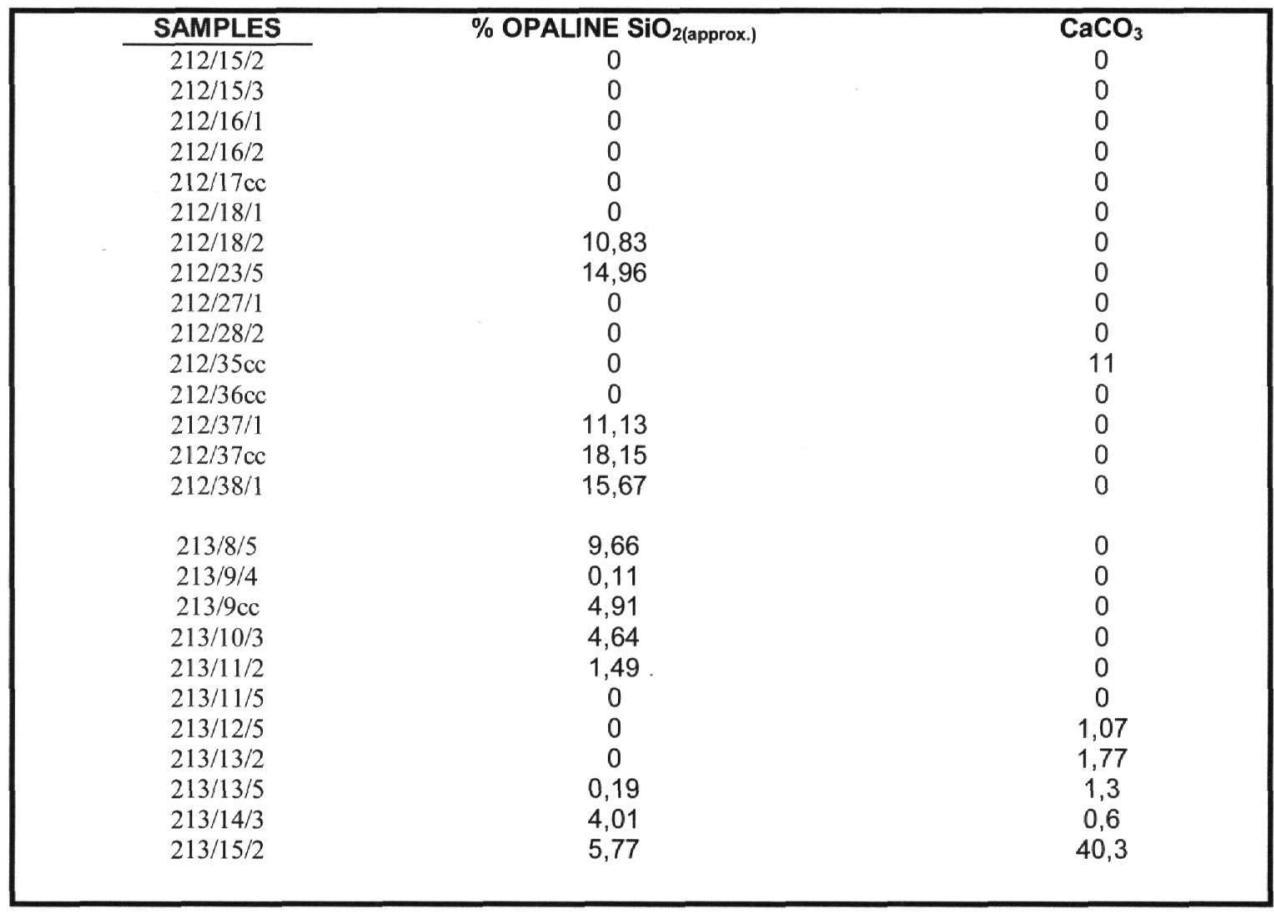

The bulk rock mineralogy data are presented here are originated from the work of the Shipboard Scientific Party of Leg.22 (Kolla 1974, Matti et al. 1974) and were also calculated on a semiquantitative basis.

\section{Results}

\subsection{Bulk Mineralogy}

The results of the bulk and clay mineralogical are presented in Tables 3 and 4 respectively.

Comparing the calcite percentages obtained from the method proposed by Weijden et al. (2006), presented in Table 2 with calcite percentages derived from the Shipboard Scientific Party of Leg.22 listed in Table 3 it is obvious that the two values are very close.

It is clear from the bulk mineralogical results (Table 3 ) that clays is the dominant phase in the samples from Site 212 giving values often more than $60 \%$. Quartz and Plagioclase and KFeldspars (of terrigenous origin) are the other more abundant phases whilst the Calcite content in most of the samples (except 18/2 and $35 \mathrm{cc}$ ) is negligible.

In Site 213 clay is the most abundant phase along with quartz, while K-Feldspars and Plagioclase are most probably detrital and to be the rest most important phases. Calcite is also absent with one exception (sample 15/2) where its content is around $41 \%$.

Mainly in Site 212 and less in Site 213 the opaline silica (opal-CT) phase was detected (irregular structural interstratification of cristobalite and tridymite layers) which is reflected in the chemical analyses (Tables 3,5 ) with enhanced percentages of $\mathrm{SiO}_{2}$. In marine sediments these phases are usually authigenic of biogenous origin (Chester 1990). 
Table 3 - Bulk rock mineralogy(on a semi-quantitative basis; data originated from the work of the Shipboard Scientific Party of Leg.22 (Kolla 1974, Matti et al. 1974)

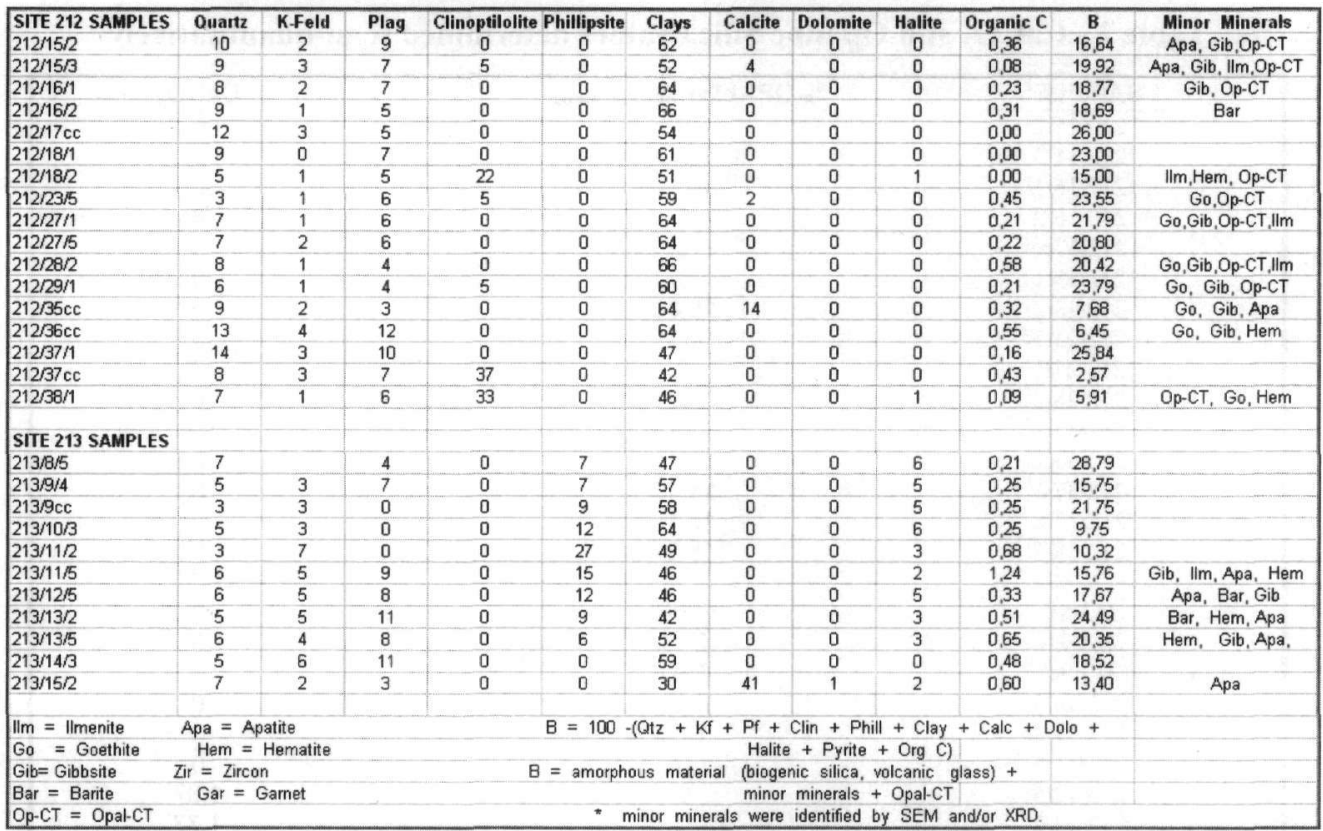

Two important zeolitic minerals were detected in this study. In Site 212 Clinoptilolite $(\mathrm{Na}, \mathrm{K})_{3.6}$ $\mathrm{Al}_{3.6} \mathrm{Si}_{14.4} \mathrm{O}_{36} .8 .8 \mathrm{H}_{2} \mathrm{O}$ was detected in various samples with considerable amounts (Table 3 ). On the other hand in Site 213 Phillipsite $\left[(\mathrm{K}, \mathrm{Na}, \mathrm{Ca})_{2}(\mathrm{Si}, \mathrm{Al})_{8} \mathrm{O}_{16} \cdot 6 \mathrm{H}_{2} \mathrm{O}\right]$ is present in considerable amounts in most of the samples reaching values up to $27 \%$. In most of the cases both zeolites in deep sea sediments are authigenic (Chester 1990, Cosgrove and Papavassiliou 1979).

\subsection{Clay Mineralogy}

Clay Mineralogy was carried out in the Institute de Mineralogie of the University of Leige. The $<2$ $\mu \mathrm{m}$ fraction was examined by $\mathrm{X}$ ray diffraction technique. A first run was completed in routine through three usual tests (air dried, solvated with ethylene glycol vapours, heated to $500{ }^{\circ} \mathrm{C}$ ). Afterwards some samples were subjected to postreatment (Gationic saturations) in order to achieve a more accurate identification particularly at the level of swelling clay components.

Oriented mounts for X-R-Diffraction were prepared leaving dilute aqueous suspensions of the $<2$ $\mu \mathrm{m}$ fraction to dry in air on glass slides. X-ray diffractograms were obtained using a Philips diffractometer with L-ka radiation. Semiquantitative determinations were carried out by using the techniques applicable by J.THOREZ in the Institut De Mineralogie in the Urersity of Liege.

The results of this semiquantitative analysis are presented in Table 4.

The expandable mixed layerd Smectite/Illite is the most abundant clay minerals phase concerning the clay mineralogy results reaching values up to $90 \%$ in both Sites. This phase seems to have originated from two sources. The most important is authigenic. In the central Indian Ocean, smectite-rich clays appear to have derived mainly from the alteration of in situ submarine basalts and the associated volcanic products (Griffin et al. 1968, Kolla et al. 1976). This suggestion is confirmed by the fact that in both Sites (212 and 213) the basement drilled was metabasalt with limestone inclusions in Site 212 and basalt in Site 213 (Sclater et al. 1974, Subbaro et al. 1979).

The other source for smectites and the expandable layerd smectite/illite is detrital. According to Kolla et al. (1973) the Quaternary smectite-rich province of Indonesia with higher abundance of smectite adjacent to the Indonesian Islands, on the Cocos-Roo Rises and on the Ninety-east Ridge 
between $5^{\circ} \mathrm{N}$ and $14^{\circ} \mathrm{S}$ can be an important source. This province is influenced by aeolian transport of silicic volcanic ash from the Indonesian island arc.

Table 4 - Clay Mineralogy results

\begin{tabular}{|c|c|c|c|c|c|}
\hline $\begin{array}{l}\text { Sample } \\
\text { No. }\end{array}$ & $\begin{array}{l}\text { Mixed } \\
\text { Layer } \\
\text { Illite/Smec } \\
\text { tite }\end{array}$ & Illite & Kaollnite & Chlorite & Palygorskite \\
\hline $212 / 15 / 2$ & 54 & 28 & 10 & 5 & 2 \\
\hline $212 / 16 / 1$ & 74 & 11 & 15 & & \\
\hline $212 / 17 / c \mathrm{cc}$ & 73 & 22 & 5 & & \\
\hline $212 / 18 / 1$ & 78 & 21 & 1 & & \\
\hline $212 / 18 / 2$ & 88 & 9 & 1 & & 2 \\
\hline $212 / 23 / 5$ & 91 & 9 & & & \\
\hline $212 / 27 / 1$ & 51 & 27 & 13 & 4 & 5 \\
\hline $212 / 27 / 5$ & 70 & 6 & 24 & & \\
\hline $212 / 28 / 2$ & 82 & 5 & 13 & & \\
\hline $212 / 29 / 1$ & 78 & 3 & 19 & & \\
\hline $212 / 35 \mathrm{cc}$ & 73 & 19 & 8 & & \\
\hline $212 / 36 \mathrm{cc}$ & 43 & 36 & 15 & 6 & \\
\hline $212 / 37 / 1$ & 83 & 10 & 3 & & 4 \\
\hline $212 / 37 \mathrm{cc}$ & 65 & 27 & & & 8 \\
\hline $212 / 38 / 1$ & 45 & 35 & & & 20 \\
\hline $213 / 8 / 5$ & 83 & 8 & 3 & 5 & \\
\hline $213 / 9 / 4$ & 96 & 2 & 2 & & \\
\hline $213 / 9 c c$ & 92 & 4 & 4 & & \\
\hline $213 / 10 / 3$ & 90 & 6 & 4 & & \\
\hline $213 / 11 / 2$ & 89 & 6 & 5 & & \\
\hline $213 / 11 / 5$ & 84 & 10 & 6 & & \\
\hline $213 / 12 / 5$ & 77 & 18 & 5 & & \\
\hline $213 / 13 / 2$ & 74 & 22 & 4 & & \\
\hline $213 / 13 / 5$ & 74 & 20 & 4 & & 2 \\
\hline $213 / 14 / 3$ & 77 & 14 & 2 & 2 & 5 \\
\hline $213 / 15 / 2$ & 83 & 11 & 6 & & \\
\hline
\end{tabular}

The other clay minerals 'i.e Kaolinite, Illite/Chlorite have a detrital origin. The relatively high percentages of Kaolinite and Illite in the clay fraction off the coast of western and north west Australia, southern Wharton Basin and on the Ninetyeast Ridge, has an aeolian origin from western Australia (Griffin et al. 1968, Kolla et al. 1981).

One important aspect in the clay mineralogy is the presence of Palygorskite mainly in a number of samples from Site 212 and to a lesser extent in a few samples from Site 213 (Table 4).

Palygorskite a Mg-rich aluminosilicate $(\mathrm{Mg}, \mathrm{Al})_{2} \mathrm{Si}_{4} \mathrm{O}_{10}(\mathrm{OH}) \cdot 4\left(\mathrm{H}_{2} \mathrm{O}\right)$ in deep sea sediments may have a detrital or authigenic origin. However its origin (at least for the most of it) in both Sites and mainly in Site 212 seems to be authigenic. As pointed out by Weser (1974) its association with the authigenic minerals smectite-Clinoptilolite and opal-CT implies an authigenic origin.

\subsection{Chemical analyses results}

The chemical analyses of bulk samples are presented in Table 5.

In order to normalize the values for the non-biogenic sediments (i.e removing biogenic carbonate and/or opal) values of $\mathrm{Ti}, \mathrm{Mg}, \mathrm{Fe}, \mathrm{K}$, and $\mathrm{Mn}$ are commonly reported as the atomic ratios $\mathrm{Ti} / \mathrm{Al}$, Fe/Al etc. (Donnelly and Wallace 1976, Weijden et al. 2006). In order to show more clearly the presence of opaline silica in the samples the $\mathrm{Si} / \mathrm{Al}$ atomic ratio was also calculated.

In Table 6 these atomic ratios from the analysed samples are shown, with their averages whilst in Table 7 the average chemical composition of some important marine sediments and igneous rocks with their atomic ratios are shown for comparisons. 

elements in ppm)

\begin{tabular}{|c|c|c|c|c|c|c|c|c|c|c|c|c|c|c|c|c|c|c|c|c|c|c|c|c|c|c|c|c|}
\hline \multicolumn{2}{|c|}{ SITE 212 SAMPLES \$i } & \multirow{2}{*}{$\frac{\pi i}{0.53}$} & \multirow{2}{*}{$\frac{\text { Al }}{9,04}$} & \multicolumn{2}{|c|}{$\mathrm{Fe}_{\text {flowat }} \mathrm{Mg}$} & \multirow{2}{*}{$\frac{\mathrm{Ca} N}{0,79}$} & \multirow{2}{*}{$\frac{\mathrm{Na}}{1,71}$} & \multirow{2}{*}{$\frac{K \quad F}{2.23}$} & \multirow{2}{*}{$\frac{P}{0,18}$} & \multirow{2}{*}{$\frac{\text { L.O.L. }}{9,48}$} & \multirow{2}{*}{$\frac{\mathrm{CO}_{2} \mathrm{l}}{1,31}$} & \multirow{2}{*}{$\frac{\text { Min }}{2.30}$} & \multirow{2}{*}{$\frac{V}{212}$} & \multirow{2}{*}{$\frac{C}{97}$} & \multirow{2}{*}{$\frac{\mathrm{Ni}}{372}$} & \multirow{2}{*}{$\frac{\text { CII }}{294}$} & \multirow{2}{*}{$\frac{\mathrm{Zn}}{1+0}$} & \multirow{2}{*}{$\frac{\text { As }}{41}$} & \multirow{2}{*}{$\frac{\mathbf{R b}}{95}$} & \multirow{2}{*}{$\frac{\mathrm{Sr}}{425}$} & \multirow{2}{*}{$\frac{Y}{92}$} & \multirow{2}{*}{$\frac{z x}{210}$} & Nb & Mo & Ba & La & $\mathrm{Ce}_{\mathrm{e}}$ & Pb \\
\hline 212152 & 22,30 & & & 8,22 & 1,96 & & & & & & & & & & & & & & & & & & 20 & 78 & 680 & 93 & 143 & 8 \\
\hline 212153 & 21,58 & 0,49 & 8.52 & 8.67 & $1, \pi$ & 1.83 & 1.51 & 2,14 & 0,16 & 9.58 & 2,30 & 2.26 & 211 & 85 & 365 & 291 & 131 & 40 & 90 & 473 & B4 & 190 & 17 & ${ }^{4} 4$ & 529 & 88 & 122 & 7 \\
\hline 212161 & 22,99 & 0,58 & 9,36 & 8,56 & 1,76 & 0,74 & 0.34 & 2.18 & 0,15 & 10.65 & 1,64 & 1.87 & 225 & 87 & 337 & 240 & 16: & 35 & 98 & 418 & 82 & 185 & 15 & 19 & 634 & 120 & 137 & 56 \\
\hline 212162 & 22,85 & 0.50 & 9,41 & 7,85 & 1,59 & 0.67 & 0,19 & 1,92 & 0,11 & 1392 & 1,90 & 1.24 & 244 & 300 & 311 & 254 & 142 & 4 & 99 & 425 & 84 & 193 & 14 & 46 & 365 & 100 & 155 & 62 \\
\hline $21217 \mathrm{ce}$ & 23.01 & 0.55 & 8.76 & 9,40 & 1,72 & 0.82 & 1,19 & 2.56 & 0.21 & 8.58 & 0,90 & 192 & 281 & 65 & 239 & 193 & 174 & 57 & 97 & 553 & 82 & 184 & 12 & 59 & 792 & 76 & 153 & S4 \\
\hline 212181 & 23,06 & 0.51 & 9,46 & 9,04 & 1,79 & 0,61 & 1,47 & 1,99 & 0,14 & 8.50 & 0,66 & 169 & 260 & 59 & 204 & 188 & 163 & 56 & 9 & +00 & 69 & 186 & 10 & 45 & 595 & 115 & 127 & 56 \\
\hline 212182 & 26,17 & 0.51 & 7,97 & 6,49 & 2,04 & 0,61 & 1,72 & 2,57 & 0.07 & 7,50 & 1,02 & 0.40 & 129 & 60 & 92 & 113 & 123 & 23 & 82 & 545 & +4 & 139 & II & 5 & 741 & 4 & 126 & 33 \\
\hline 2122235 & 26,85 & 0.36 & 7,49 & 7,61 & 2.59 & 0,66 & 1,92 & 1.90 & 0.07 & 7,00 & 2.33 & 0.16 & 162 & 133 & 103 & 55 & 131 & Bd & 82 & 237 & to & 109 & 10 & 0 & 348 & 7 & 9: & 16 \\
\hline $212 / 271$ & 21,40 & 0.56 & 12,90 & 8,10 & 1,27 & 0.61 & 1,31 & 1.88 & 0.17 & 9,03 & 0.88 & 0.23 & 186 & 93 & 91 & 161 & 181 & 42 & 90 & 197 & 3 & 159 & II & 13 & 688 & 119 & 161 & 48 \\
\hline 212282 & 21,93 & 0.50 & 13,34 & 7,71 & 1,32 & 0,59 & 1.22 & 1.61 & 0,15 & 10.00 & 212 & 0.51 & 224 & 178 & 144 & 112 & 145 & 30 & 79 & 200 & 62 & 154 & 17 & 29 & 519 & 116 & 174 & 40 \\
\hline $21236 \mathrm{cc}$ & 21,68 & 0,44 & 8,62 & 5.65 & 1,66 & 5,62 & 1,34 & 2,30 & 0,09 & 12,55 & 9,43 & 0,17 & 141 & 94 & 89 & 151 & 136 & 12 & 90 & 273 & 45 & 122 & 9 & 21 & 622 & 42 & 126 & 34 \\
\hline $21236 \mathrm{Cc}$ & 26,30 & 0,52 & 9,90 & 6.98 & 1,63 & 0,46 & 0.67 & 3,02 & 0,09 & 788 & 2.50 & 0,12 & 132 & 118 & 122 & 102 & 158 & 17 & 131 & 147 & 53 & 174 & 14 & 9 & 723 & 86 & $1+4$ & 38 \\
\hline 212371 & 27,05 & 0,58 & 8,25 & 5,70 & 2,11 & 0,50 & 1,13 & 2,91 & 0,07 & 7.23 & 0,60 & 0,35 & 103 & 69 & 91 & 187 & 145 & 0 & 116 & 155 & 45 & 199 & 14 & 7 & 638 & 101 & 174 & 50 \\
\hline $21237 \mathrm{cc}$ & 27,92 & 0,46 & 7,34 & 4,94 & 1,96 & 0,66 & 1.66 & 2.60 & 0,11 & 7.8 & 2,27 & 0,70 & 77 & 48 & 117 & 129 & 158 & 9 & 96 & 329 & $\infty$ & 147 & 8 & 0 & 701 & 65 & 136 & 34 \\
\hline 312381 & 27,36 & 0.53 & 7.56 & 5.34 & 1,95 & 0.64 & 1.51 & 2.69 & 0,10 & 783 & 1.04 & 0,50 & 86 & 48 & 84 & 157. & 149 & 7 & 97 & 316 & 58 & 169 & 10 & 4 & 916 & 68 & 155 & 37 \\
\hline STTE 213 SAMI & & & & & & & & & & & & & & & & & & & & & & & & & & & & \\
\hline 21385 & 26,71 & 0,32 & 8,38 & 3,70 & 1,26 & 0,61 & 3,26 & 1,98 & 0,07 & 9,18 & 0,76 & 062 & 73 & 72 & 206 & 182 & 0 & 11 & 81 & 108 & 58 & 136 & 6 & 16 & 324 & 55 & 48 & 31 \\
\hline 21394 & 24.20 & 0,41 & 9,11 & 5.41 & 2,13 & 0.99 & 3,35 & 1,62 & 0,17 & 8,75 & 0,90 & 1,10 & 124 & 159 & 24 & 291 & no & 15 & 83 & 143 & 110 & 174 & 9 & 28 & 590 & 101 & 119 & 43 \\
\hline $2139 \mathrm{cc}$ & 24,35 & 0,30 & 8,33 & 4,62 & 2.27 & 0,99 & 3,44 & 1.43 & 0.26 & 10.20 & 0.90 & 1.30 & 74 & 145 & 277 & 329 & 116 & 17 & 64 & 175 & 152 & 154 & 9 & 49 & 240 & 99 & 63 & 43 \\
\hline 213103 & 24,49 & 0.29 & 8,43 & 4,40 & 2,18 & 0,97 & 3,14 & 1,45 & 0.25 & 9,43 & 0.90 & 1.60 & 96 & 26 & 320 & 346 & 102 & 13 & 55 & 151 & 129 & 138 & 0 & 32 & $38 \mathrm{H}$ & 86 & 95 & 4 \\
\hline 213112 & 23.87 & 0.28 & 8,75 & 4,67 & 1,85 & 0,96 & 3,52 & 2,70 & 0,27 & 8,68 & 2,51 & 194 & 93 & 91 & 418 & 335 & 102 & 15 & 73 & 129 & 121 & 133 & 11 & +4 & 326 & 88 & 119 & 45 \\
\hline 2134115 & 22,33 & 0.39 & 8,82 & 6.34 & 1,79 & 1.12 & 2,69 & 2.42 & 0.36 & 9.63 & 4.55 & 2.35 & 147 & 59 & 606 & 372 & $14^{7}$ & 29 & 83 & 178 & 155 & 169 & 15 & 67 & 426 & 129 & $1+6$ & 55 \\
\hline 213125 & 21,83 & 0,50 & 8,32 & 7,43 & 1,88 & 1,58 & 3,23 & 2.57 & 0,44 & 9,55 & 1,21 & 2,17 & 162 & $5:$ & +62 & $2+1$ & 145 & 31 & 75 & 197 & 172 & 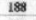 & 15 & to & 458 & 161 & $1+1$ & 49 \\
\hline $21313 / 2$ & 22,13 & 0,47 & 8,52 & 7.14 & 1,86 & 1,89 & 2,74 & 2,71 & 0,55 & 8.50 & 1,87 & 2,32 & 175 & 61 & 479 & 280 & 170 & w3 & $\pi$ & 196 & 229 & 191 & 13 & 41 & $399^{\circ}$ & 126 & 131 & 49 \\
\hline 213135 & 22,37 & 0,56 & 8.41 & 7.43 & 2,07 & 1,68 & 2.30 & 2,43 & 0,47 & 893 & 2,31 & 2.16 & 188 & 43 & 416 & 234 & 19 & 30 & 0 & 0 & 0 & 0 & 0 & 0 & 538 & 169 & 157 & 52 \\
\hline 21303 & 23,97 & 0,50 & 8,34 & 6,47 & 2,58 & 1,39 & 1,84 & 2,82 & 0,40 & 8.28 & 1,0 & 0.82 & 121 & 53 & 229 & 134 & 143 & 7 & 88 & 144 & 117 & 163 & 15 & 19 & 513 & 141 & 152 & 40 \\
\hline $213 / 5 / 2$ & 13,33 & 0.28 & 4,01 & 6,71 & 1,38 & 16,71 & 1,22 & 1,95 & 0,33 & 22,18 & 18.60 & 081 & 137 & 170 & 131 & 125 & 116 & 43 & $4^{7}$ & 352 & 62 & $10^{7}$ & 16 & 11 & 324 & 102 & 77 & 44 \\
\hline
\end{tabular}

Table 6 - Critical Chemical ratios for the under study samples from Sites 212 and 213

\begin{tabular}{|c|c|c|c|c|c|c|c|c|c|c|c|}
\hline SAMPLES & Si/AI & TIIAI & $\mathrm{Mg} / \mathrm{Al}$ & $\mathrm{Fe} / \mathrm{Al}$ & $\mathrm{Na} / \mathrm{Al}$ & KIAI & Cr/Al & Ni/A & $\mathrm{Mn} / \mathrm{Al}$ & $\mathrm{Fe} / \mathrm{Mn}$ & $\begin{array}{c}\text { DEPTH } \\
\text { BELOW SEA } \\
\text { FLOOR(m.) }\end{array}$ \\
\hline $212 / 15 / 2$ & 2,369 & 0,033 & 0,241 & 0,439 & 0,222 & 0,090 & 0,00056 & 0,00189 & 0,12494 & 3,573 & 290 \\
\hline $212 / 15 / 3$ & 2,432 & 0,032 & 0,231 & 0,491 & 0,207 & 0,091 & 0,00052 & 0,00197 & 0,13024 & 3,834 & 292 \\
\hline $212 / 16 / 1$ & 2,359 & 0,035 & 0,208 & 0,442 & 0,043 & 0,085 & 0,00048 & 0,00165 & 0,09813 & 4,578 & 298,5 \\
\hline $212 / 16 / 2$ & 2,332 & 0,030 & 0,188 & 0,401 & 0,024 & 0,074 & 0,00110 & 0,00152 & 0,09601 & 4,250 & 300 \\
\hline 212/17cc & 2,523 & 0,035 & 0,219 & 0,518 & 0,159 & 0,106 & 0,00038 & 0,00125 & 0,10764 & 4,896 & 309 \\
\hline $212 / 18 / 1$ & 2,342 & 0,030 & 0,210 & 0,462 & 0,182 & 0,076 & 0,00032 & 0,00099 & 0,08774 & 5,351 & 317,5 \\
\hline $212 / 18 / 2$ & 3,155 & 0,036 & 0,284 & 0,391 & 0,254 & 0,117 & 0,00039 & 0,00053 & 0,02466 & 16,139 & 319 \\
\hline $212 / 23 / 5$ & 3,441 & 0,026 & 0,383 & 0,485 & 0,301 & 0,092 & 0,00092 & 0,00063 & 0,01048 & 47,035 & 371 \\
\hline $212 / 27 / 1$ & 1,593 & 0,025 & 0,109 & 0,301 & 0,119 & 0,053 & 0,00037 & 0,00032 & 0,00875 & 34,970 & 403 \\
\hline $212 / 28 / 2$ & 1,579 & 0,021 & 0,110 & 0,278 & 0,107 & 0,044 & 0,00069 & 0,00050 & 0,01878 & 15,030 & 413,8 \\
\hline $212 / 35 \mathrm{cc}$ & 2,416 & 0,029 & 0,214 & 0,311 & 0,182 & 0,097 & 0,00057 & 0,00047 & 0,00969 & 32,666 & 485,5 \\
\hline $212 / 36 \mathrm{cc}$ & 2,455 & 0,029 & 0,183 & 0,280 & 0,079 & 0,111 & 0,00062 & 0,00057 & 0,00595 & 47,792 & 489 \\
\hline $212 / 37 / 1$ & 3,150 & 0,039 & 0,284 & 0,331 & 0,160 & 0,128 & 0,00043 & 0,00051 & 0,02083 & 16,146 & 497,8 \\
\hline $212 / 37 \mathrm{cc}$ & 3,655 & 0,035 & 0,297 & 0,325 & 0,266 & 0,128 & 0,00034 & 0,00073 & 0,04686 & 7,054 & 498,5 \\
\hline $212 / 38 / 1$ & 3,475 & 0,039 & 0,287 & 0,339 & 0,234 & 0,129 & 0,00033 & 0,00051 & 0,03246 & 10,631 & 507,5 \\
\hline AVERAGE & & & & & & & & & & & \\
\hline $\begin{array}{l}\text { SITE } 212 \\
\text { VALUES }\end{array}$ & 2,618 & 0,032 & 0,230 & 0,386 & 0,169 & 0,095 & 0,00054 & 0,00094 & 16,92964 & 16,930 & \\
\hline $213 / 8 / 5$ & 3,062 & 0,021 & 0,167 & 0,212 & 0,457 & 0,086 & 0,00045 & 0,00113 & 0,03634 & $5,934 €$ & 73 \\
\hline $213 / 9 / 4$ & 2,550 & 0,025 & 0,260 & 0,287 & 0,432 & 0,064 & 0,00091 & 0,00123 & 0,05927 & $4,921 \in$ & 81 \\
\hline $213 / 9 c c$ & 2,809 & 0,020 & 0,294 & 0,268 & 0,485 & 0,062 & 0,00090 & 0,00153 & 0,07668 & $3,556 €$ & 84,5 \\
\hline $213 / 10 / 3$ & 2,792 & 0,020 & 0,287 & 0,252 & 0,437 & 0,063 & 0,00016 & 0,00175 & 0,09325 & $2,750 €$ & 89,5 \\
\hline $213 / 11 / 2$ & 2,621 & 0,018 & 0,235 & 0,258 & 0,472 & 0,112 & 0,00054 & 0,00220 & 0,10889 & $2,408 €$ & 97 \\
\hline $213 / 11 / 5$ & 2,431 & 0,025 & 0,225 & 0,347 & 0,357 & 0,100 & 0,00035 & 0,00316 & 0,13079 & $2,696 €$ & 101,5 \\
\hline $213 / 12 / 5$ & 2,521 & 0,034 & 0,251 & 0,432 & 0,455 & 0,112 & 0,00032 & 0,00255 & 0,12815 & $3,423 €$ & 110,8 \\
\hline $213 / 13 / 2$ & 2,494 & 0,031 & 0,242 & 0,405 & 0,378 & 0,116 & 0,00037 & 0,00258 & 0,13369 & $3,078 \in$ & 116,4 \\
\hline $213 / 13 / 5$ & 2,555 & 0,038 & 0,273 & 0,427 & 0,321 & 0,105 & 0,00027 & 0,00227 & 0,12612 & $3,442 €$ & 120,5 \\
\hline $213 / 14 / 3$ & 2,760 & 0,034 & 0,343 & 0,375 & 0,259 & 0,123 & 0,00033 & 0,00126 & 0,04827 & $7,890 €$ & 126,8 \\
\hline $\begin{array}{l}213 / 15 / 2 \\
\text { AVERAGE }\end{array}$ & 3,190 & 0,040 & 0,380 & 0,808 & 0,356 & 0,176 & 0,00220 & 0,00150 & 0,09914 & $8,281 €$ & 135,4 \\
\hline $\begin{array}{l}\text { SITE } 213 \\
\text { VALUES }\end{array}$ & 2,708 & 0,028 & 0,269 & 0,370 & 0,401 & 0,102 & 0,00062 & 0,00192 & 0,09460 & 4,398 & \\
\hline
\end{tabular}

\subsection{General Interpretation}

\subsubsection{Chemical characteristics of the sediments from Sites 212 and 213}

In general the average $\mathrm{Si} / \mathrm{Al}$ ratio for the analyzed samples from Site $212(2,61)$ is lower compared to the average values for terrigenous sediments, deep sea clays, shale and average continental crust. (Table 6). The same applies for the average values from Site 213 which are slightly higher compared with the average $\mathrm{Si} / \mathrm{Al}$ ratios of Site 212 . However as it can be seen from Tables 2 and 5 and Figures 2 and 3, samples rich in opaline silica exhibit values well above 3.1 especially in samples close to the basement in Site 212 core. In contrast, in Site 213 samples the presence of opaline silica is negligible with one exception.

The ratio of Ti/AI is of particular interest in the geochemistry of deep-sea sediments. Chester (1965), Chester and Aston (1976) suggested that high Ti contents strongly indicates the presence 
of basaltic pyroclastics or generally speaking the influence of basic igneous rocks. According to Couture (1977), sediments having Ti/Al ratio of 0,047 , a value being half way between that of average ocean tholeiite $(0,062)$ and average pelagic sediments $(0,031)$, could be composed of altered basaltic ash and terrigenous sediments in equal proportion. Although the average $\mathrm{Ti} / \mathrm{Al}$ ratio for Site 212 sediments is very similar to the average ratios of terrigenous sediments, shale and deep sea clays (Table 7), samples from the lower part of Site $212(37 / 1,37 \mathrm{cc}, 38 / 1)$ show considerably higher values (between $0,036-0,0393$ ). This is clearly shown in the plot of $\mathrm{Ti} / \mathrm{Al}$ versus depth (Fig. 2). So it seems quiet possible that these samples have substantial elemental input from alteration of the underlying basaltic basement. The average Ti/Al ratio for Site 213 samples is slightly lower compared with those of terrigenous marine sediments, shale and deep sea clays (Table 7). However, the samples from the lower part of the core shows similar tendency as 212 basement samples. They have considerably higher values compared with average ratios of terrigenous marine sediments, shale and deep sea clays (Tables 6, 7 and Fig. 3). Also in this case it seems that these samples have an important input from altered basalts derived from the basaltic basement underneath.

$\mathrm{The} \mathrm{Mg} / \mathrm{Al}$ ratio shows also some important characteristics (Figs 2, 3). In Site 212 the $\mathrm{Mg}$ content is controlled mainly by authigenic phases i.e zeolites and clay minerals (mixed layer Smectite/IlliTe and Palygorskite). This has been confirmed by the Factor analysis as it will be discussed below. For the samples of this Site the average $\mathrm{Mg} / \mathrm{Al}$ ratio $(0,23)$ is slightly higher when compared with average marine terrigenous matter $(0,216)$ and shale $(0,186)$ but considerably lower than the average values of deep sea clays $(0,277)$ and continental crust $(0,42)$. However sample $23 / 5$ and the samples from the lower part of the core $(37 / 1,37 \mathrm{cc}, 38 / 1)$ show the highest value in the $\mathrm{Mg} / \mathrm{Al}$ ratio (Fig. 2).

Table 7 - Average chemical composition with their critical chemical ratios for important sedimentary and igneous rocks associated with marine environment

\begin{tabular}{|c|c|c|c|c|c|c|c|c|}
\hline ELEMENTS & $\begin{array}{c}\text { AVERAGE SITE } \\
212 \\
\text { COMPOSITION }\end{array}$ & $\begin{array}{c}\text { AVERAGE SITE } \\
213 \\
\text { COMPOSITION }\end{array}$ & $\begin{array}{c}\text { AVERAGE } \\
\text { TERRIGENOUS } \\
\text { MATTER }^{1}\end{array}$ & $\begin{array}{l}\text { AVERAGE } \\
\text { DEEP SEA } \\
\text { CLAYS }^{2}\end{array}$ & $\begin{array}{l}\text { AVERAGE } \\
\text { SHALE }^{3}\end{array}$ & $\begin{array}{c}\text { AVERAGE } \\
\text { CONTINENTAL } \\
\text { CRUST }^{4}\end{array}$ & $\begin{array}{l}\text { AVERAGE } \\
\text { OCEANIC } \\
\text { CRUST }^{5} \\
\text { (MEAN } \\
\text { OCEANIC } \\
\text { BASALT) }\end{array}$ & $\begin{array}{l}\text { AVERAGE } \\
\text { MAFIC } \\
\text { IGNEOUS } \\
\text { ROCKS }^{6}\end{array}$ \\
\hline $\mathrm{SiO}_{2}$ & 51.55 & 48.54 & 53.3 & 53,5 & 50.93 & 57.3 & 47.85 & 51.36 \\
\hline Al & 9,16 & 8,13 & 8.1 & 8,4 & 8 & 8.42 & 8.11 & 8.8 \\
\hline $\mathbf{T i}$ & 0,51 & 0.39 & 0.48 & 0,46 & 0,45 & 0,54 & 1 & 0.9 \\
\hline $\mathrm{Fe}$ & 7,24 & 5,85 & 4,9 & 6,5 & 4,7 & 7,07 & 7,6 & 8,6 \\
\hline$M n$ & 1.00 & 1,563 & 0,088 & 0,67 & 0,085 & 0,14 & 0,13 & 0,2 \\
\hline Mg & 1.81 & 1,93 & 1,58 & 2,1 & 1,34 & 3,19 & 3,96 & 4,5 \\
\hline $\mathrm{Na}$ & 1.26 & 2,79 & 1.095 & 4 & 0.66 & 2,3 & 2,11 & 1,9 \\
\hline $\mathbf{K}$ & 2.30 & 2.19 & 2,25 & 2.5 & 2.3 & 0,91 & 0,72 & 0.8 \\
\hline $\mathbf{B a}$ & 0,0649 & 0,0412 & 0,054 & 0,23 & 0,058 & 0,025 & 0,0126 & 0,03 \\
\hline $\mathrm{Cu}$ & 0,0175 & 0,0261 & 0,0057 & 0,025 & 0.0057 & 0.0075 & 0.0071 & 0,01 \\
\hline $\mathbf{N i}$ & 0,0184 & 0,0344 & 0,009 & 0,0225 & 0,0095 & 0,0105 & 0,0107 & 0,016 \\
\hline $\mathrm{Zn}$ & 0.0149 & 0,0127 & 0.0078 & 0.0165 & 0,008 & 0.008 & 0.0098 & 0.013 \\
\hline $\mathbf{P b}$ & 0.0048 & 0.0045 & 0,0018 & 0,008 & 0.002 & 0.0008 & 0,0001 & 0,0008 \\
\hline $\mathrm{Cr}$ & 0,0096 & 0,0085 & 0,01 & 0,009 & 0,01 & 0,0185 & 0,0224 & 0,02 \\
\hline $\mathrm{Zr}$ & 0,0168 & 0,0141 & 0,019 & 0,015 & 0,02 & 0,01 & 0,014 & 0,01 \\
\hline $\overrightarrow{R b}$ & 0,0095 & 0.0066 & 0,0128 & 0,011 & 0.014 & 0.0032 & 0,0013 & 0,0045 \\
\hline Mo & 0.0027 & 0,0032 & 0.0002 & 0.0027 & 0.0002 & 0.0001 & 0.0001 & 0,0001 \\
\hline As & 0.0028 & 0.0022 & 0.0005 & 0.0013 & 0.0006 & 0.0001 & tr. & 0.0002 \\
\hline v & 0,0178 & 0.0126 & 0,013 & 0,012 & 0,013 & 0,023 & 0,0296 & 0.02 \\
\hline $\mathbf{Y}$ & 0,0065 & 0,0121 & 0,0031 & 0,009 & 0,003 & 0,002 & 0,0039 & 0,0025 \\
\hline Nb & 0,0013 & 0,0010 & 0,002 & 0,0014 & 0,002 & 0,0011 & 0,0015 & 0,002 \\
\hline $\mathbf{P}$ & 0,126 & 0,325 & 0,084 & 0,15 & 0,077 & 0,087 & 0,0001 & 0,14 \\
\hline $\mathrm{Ce}$ & 0,0142 & 0.0113 & 0,0054 & 0.0345 & 0,005 & 0.0033 & & 0,0048 \\
\hline La & 0,0087 & 0,0114 & 0,0033 & 0.0115 & 0,004 & 0.0016 & & 0,001 \\
\hline $\mathrm{sr}$ & 0.0341 & 0.0161 & & & & & & \\
\hline \multirow{2}{*}{\multicolumn{9}{|c|}{$\begin{array}{l}\text { Ca } \\
\text { RATIOS }\end{array}$}} \\
\hline & & & & & & & & \\
\hline $\mathrm{A} / / \mathrm{A} /+\mathrm{Fe}+\mathrm{Mn}$ & 0,5260 & 0,5230 & 0,619 & 0,540 & 0,626 & 0,539 & 0,512 & 0,500 \\
\hline $\mathrm{Fe} / \mathrm{Ti}$ & 14,252 & 14,987 & 10,208 & 14,130 & 10,444 & 13,093 & 7,600 & 9,556 \\
\hline Si/AI(A.R) & 2,618 & 2,708 & 2,954 & 2,859 & 2,858 & 3,055 & 2,648 & 2,620 \\
\hline Ti/Al(A.R) & 0,032 & 0,028 & 0,0334 & 0,0308 & 0,0317 & 0,0361 & 0,0695 & 0,0576 \\
\hline$M g / A l(A . R)$ & 0,230 & 0,269 & 0,2165 & 0,2775 & 0,1859 & 0,4205 & 0,5419 & 0,5675 \\
\hline Fe/AI(A.R) & 0,386 & 0,370 & 0,2922 & 0,3738 & 0,2838 & 0,4056 & 0,4527 & 0,4721 \\
\hline Cr/Al(A.R) & 0,0005 & 0,0006 & 0,0006 & 0,0006 & 0,0006 & 0,0011 & 0,0014 & 0,0012 \\
\hline KUAI(A.R) & 0,0947 & 0,1020 & 0,1917 & 0,2054 & 0,1984 & 0,0746 & 0,0613 & 0,0627 \\
\hline $\mathrm{Na} / \mathrm{A} /(\mathrm{A} . \mathrm{R})$ & 0,1693 & 0,4008 & 0,1586 & 0,5588 & 0,0968 & 0,3206 & 0,3053 & 0,2534 \\
\hline Ni/AI(A.R) & 0,0009 & 0,0019 & 0,0005 & 0,0012 & 0,0005 & 0,0006 & 0,0006 & 0,0008 \\
\hline$M n / A l(A, R)$ & 0,0500 & 0,0900 & 0,0053 & 0,0392 & 0,0052 & 0,0082 & 0,0079 & 0,0112 \\
\hline \multirow[t]{2}{*}{$\mathrm{Fe} / \mathrm{Mn}$} & 16,9296 & 4,3980 & 55,6818 & 9,7015 & 55,2941 & 50,5000 & 58,4615 & 43,0000 \\
\hline & & & $\begin{array}{l}{ }^{1} \text { Bostrom } \\
\text { et.al.1976 }\end{array}$ & ${ }^{2}$ Chester 1990 & $\begin{array}{c}{ }^{3} \text { Krauskopf } \\
1967\end{array}$ & $\begin{array}{c}{ }^{4} \text { Taylor and } \\
\text { McLennan } 1985\end{array}$ & ${ }^{5}$ Hart 1976 & $\begin{array}{c}{ }^{6} \text { Krauskopf } \\
1967\end{array}$ \\
\hline
\end{tabular}

In the case of sample $23 / 5$ the very high value of $\mathrm{Mg} / \mathrm{Al}(0,383)$ is explained by the fact that in this sample the clay fraction contains by more than $90 \%$ expandable layers of smectite/Illite (Table 4) 
which is Mg-rich mineral. On the other hand the increase in this ratio in the lower part of Site 212 is associated with high contents of palygorskite, (a Mg-rich mineral) ranging between 4-20\% (Table 4). However, the proximity of these bottom samples with the altered basaltic basement of Site 212 in combination with the increase in Ti/Al ratios in the same samples (Table 6, Fig. 2) implies also a volcanoclastic input in the sediments. For Site 213 the average value for the $\mathrm{Mg} / \mathrm{Al}$ ratio $(0,269)$ is considerably higher when compared with that of the average marine terrigenous matter $(0,216)$ and shale $(0,186)$ and very close to the average values of deep sea clays $(0,277)$. Also most of the $\mathrm{Mg} / \mathrm{Al}$ values for this Site are higher when compared with Site 212. (Table 6, Fig. 3). These higher values are associated with the fact that in contrast with Site 212, in Site 213, most of the $\mathrm{Mg}$ content is associated with the higher percentage of clay minerals (mainly expandable mixed layers Illite/Smectite) (Table 4). This has been confirmed by the factor analysis shown below. However, similar to site 212 the $\mathrm{Mg} / \mathrm{Al}$ ratio shows considerable increase with the increasing depth (Fig. 3). This partly is related to the fact that in this Site there is presence of Palygorskite and small amounts of Dolomite in the samples close to basement (Tables 3, 4). On the other hand the same trend of $\mathrm{Ti} / \mathrm{Al}$ and $\mathrm{Mg} / \mathrm{Al}$ ratios in both Sites to increase close to basaltic basement (Figs 2 and 3) and this leads to the conclusion that the volcanic activity in the area had an important contribution concerning $\mathrm{Ti}$ and $\mathrm{Mg}$. Goldschmidt (1954) mentioned that high $\mathrm{MgO}$ (3-6\%) is observed in the sediments if the original constituents of the sediments were derived from basic igneous rocks rich in $\mathrm{Mg}$ and especially when volcanic ash from basaltic, andesitic and related volcanoes were deposited with the residual and hydrolysate sediments.

The $\mathrm{Fe} / \mathrm{Al}$ and $\mathrm{Mn} / \mathrm{Al}$ ratios seem to be important. Both ratios exhibit similar trends. In Site 212 both are higher in the upper part of the core whilst there is a considerable decrease in the lower part (Table 6, Fig. 2). This trend is common in the upper part of marine pelagic sediments especially when the environment is oxidizing $\left(\mathrm{E}_{\mathrm{h}}>0\right)$ since $\mathrm{Fe}^{+2}$ and $\mathrm{Mn}^{+2}$ ions are oxidized to $\mathrm{Fe}^{+3}$ and $\mathrm{Mn}^{+4}$ forming oxides. For Site 212 samples the average value of the $\mathrm{Fe} / \mathrm{Al}$ ratio is 0,386 , which is well above terrigenous marine sediments $(0,29)$ and shales $(0,28)$ but very close to deep sea clays $(0,37)$ and average continental crust $(0,40)$ (Table 7 ). The average $\mathrm{Mn} / \mathrm{Al}$ ratio is 0,05 , which is almost ten times more than the corresponding ratio for terrigenous marine sediments, shales $(0,005)$ and average continental crust $(0,008)$ whilst it is closer to the average corresponding value for Deep Sea Clays $(0,039)$ (Table 7$)$.

Based on the above findings it is obvious that an additional source for $\mathrm{Mn}$ and partly for Fe is required in order to explain such enrichment which especially for Mn, except for the upper part of the core, where the highest values were found (Tables 5,6). This extra source for the upper part is due to the authigenic formation of $\mathrm{Mn}-\mathrm{Fe}$ oxides via diagenetic or hydrogenous processes forming a ferromanganese coating very often reported from deep sea clays (Chester and Aston 1976, Chester 1990).

For Site 213 the trends of the $\mathrm{Fe} / \mathrm{Al}$ and $\mathrm{Mn} / \mathrm{Al}$ ratios are also parallel but they follow the opposite trend compared to Site 212 samples. As it is evident from Table 6 and Fig. 3 both ratios have an impressive increase in the lower part of the core close to the basaltic basement. The average Fe/Al ratio for the Site 213 analysed samples $(0,37)$ is also well above terrigenous marine sediments $(0,29)$ and shales $(0,28)$ but very close to deep sea clays $(0,37)$ and average continental crust $(0,40)$ (Table 8 ), whilst the average $\mathrm{Mn} / \mathrm{Al}$ ratio is 0,09 almost 18 times more than the corresponding ratio for terrigenous marine sediments, shales $(0,005)$ and average continental crust $(0,008)$ and more than twice for the average corresponding value for deep sea Clays $(0,039)($ Table 7). Again it is evident that an extra source for $\mathrm{Mn}$ and partly for $\mathrm{Fe}$ is required in order to explain such enrichments which in the case of Site 213 could be related to some local hydrothermal activity related with the tectonic activity in the basement of this Site, leading to the formation of $\mathrm{Mn}-\mathrm{Fe}$ oxides in authigenic deep sea clays. 

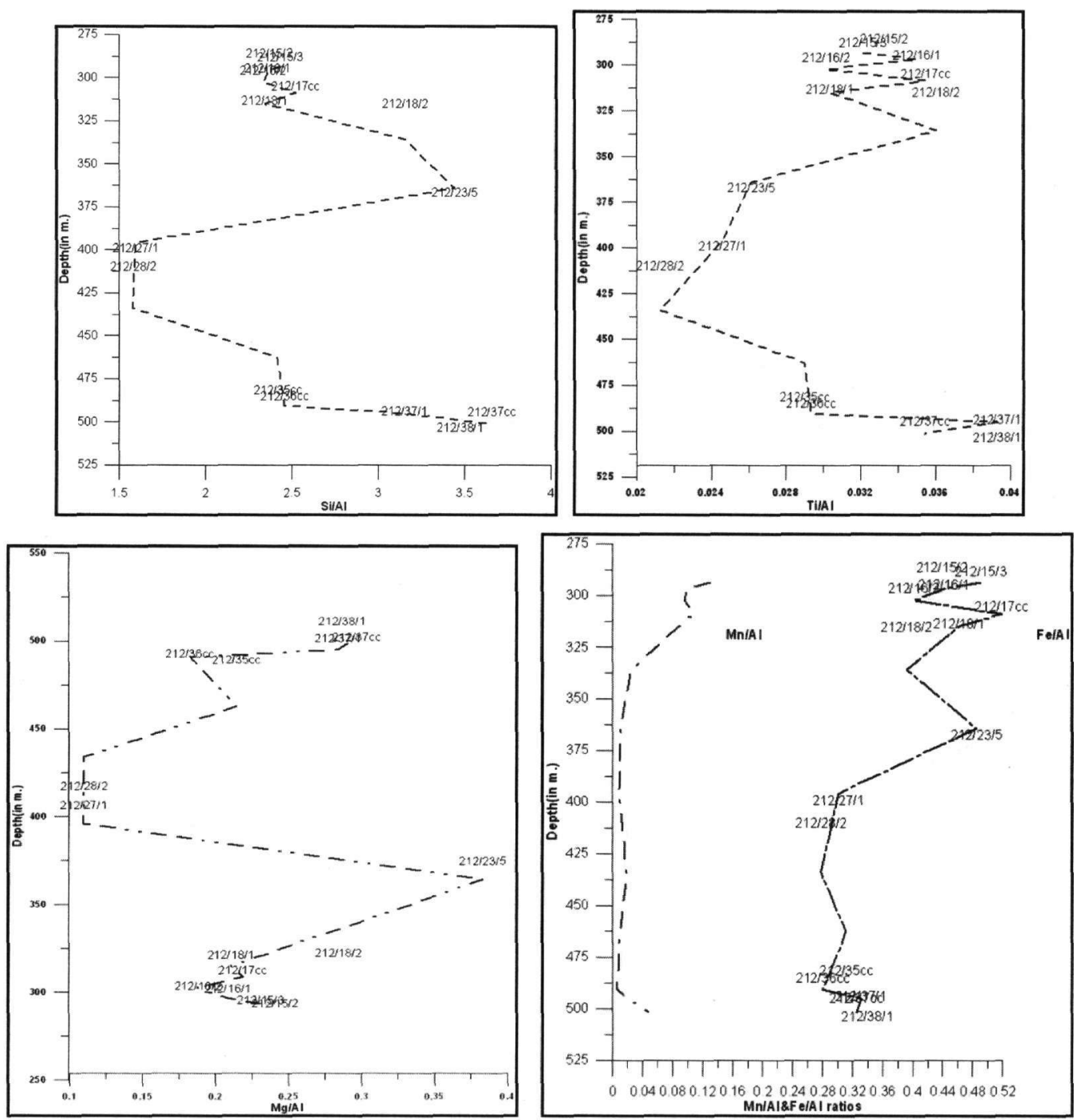

Figure 2 - Plots of the Si/Al, Ti/Al, $\mathrm{Mg} / \mathrm{Al}, \mathrm{Fe} / \mathrm{Al}$ and $\mathrm{Mn} / \mathrm{Al}$ (atomic ratios) versus depth for Site 212 samples

This is evident also from the chemical analysis where Mn values more than $2 \%$ have been recorded in both Sites. The description of the upper part of Site 212 sediments as Fe-oxide rich brown zeolitic claystone (Table 1) by the Shipboard Scientific Party of Leg.22 (Pimm 1974) confirms the above conclusion.

According to Sclater et. al. (1974) Around 100-105 m.y. B.P. (Albian - Cenomanian) a spreading centre trending slightly south of east with transform faults trending just east of, north became active in the Central Indian and Wharton Basins and for the next 20 m.y. B.P. India moved in north-north-easterly direction with respect to Antarctica. Therefore, the early Cretaceous sediments of the eastern sites such as 212 and 213 , probably received hydrothermal and volcanic input from this spreading Centre. The existence of the volcanogenic and authigenic inputs as well as the existence of the biogenous component in both 212 and 213 Sites has been confirmed by similar findings in the Broken Ridge (southern to Sites 212 and 213) during the Ocean Drilling Project at ODP Site 752A (Owen and Zimmerman 1991). 

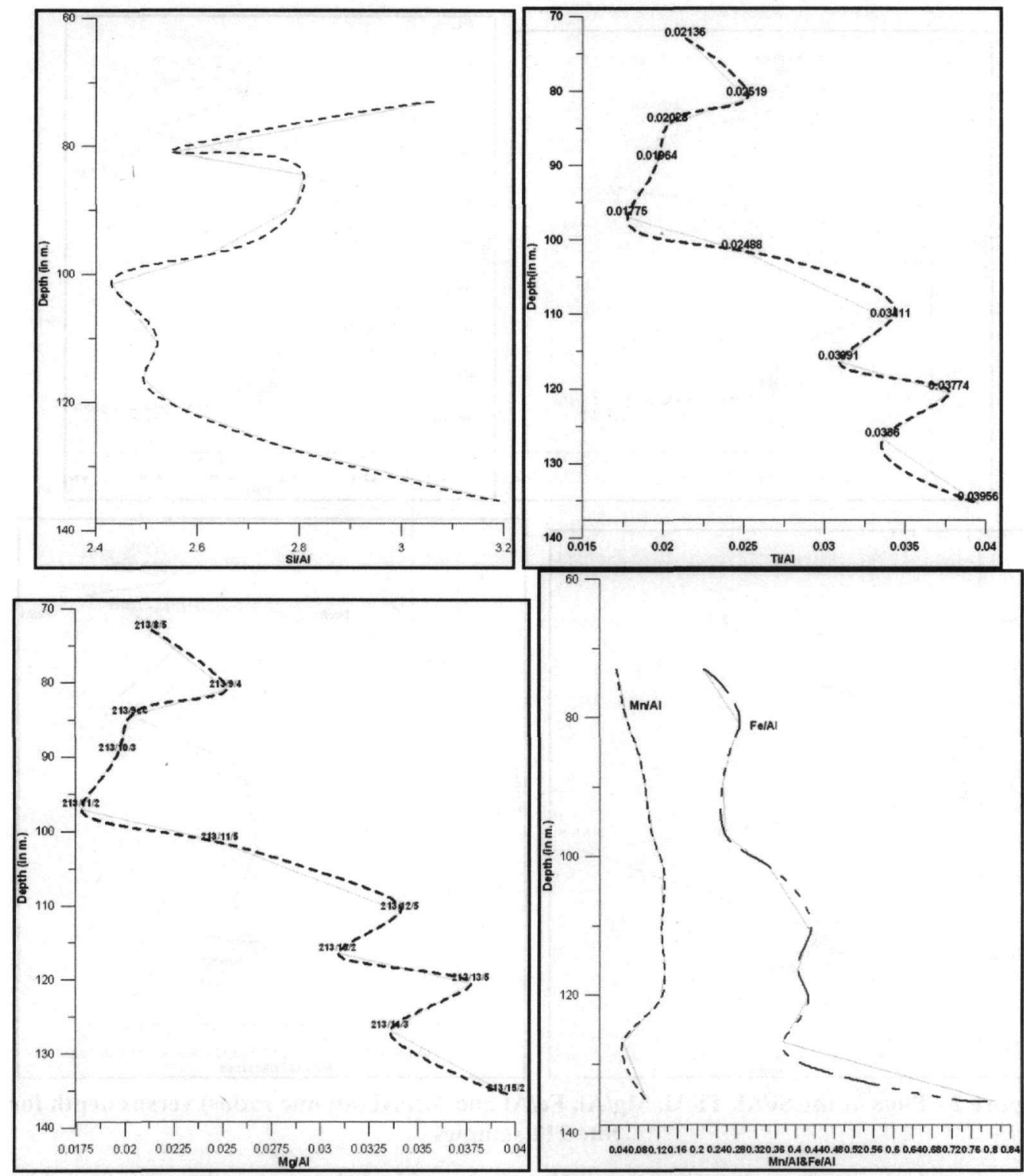

Figure 3 - Plots of the Si/Al, Ti/Al, Mg/Al, Fe/Al and Mn/Al (atomic ratios) versus depth for Site 213 samples

\subsubsection{Factor Analysis}

Factor analysis using the STATISTICA statistical programme was used in order to identify interelement groupings in an attempt to understand the geochemical nature and history of the sediments, using only the original bulk chemical analyses. Oblique varimax factors were extracted, these having the advantage of a correlation matrix between the various factors, aiding their interpretation. Factors were derived from orthogonal rotations of principal-component eigenvectors by use of the Varimax method. All communalites are $\geq 0,90$.

The statistical method of factor analysis of the total major and trace element data confirms most of the element associations deduced from the previous analysis for both Sites, and provides a summary of the inter element relationships. 


\subsubsection{Site 212}

After the principal components analysis, six factors, accounted for 90,76 of the total variance were extracted. The factor loadings for these 6 factors are shown in Table 8. Factors were derived from orthogonal rotations of principal-component eigenvectors by the use of the Varimax method. All communalites are $\geq 0,90$. Low factor loadings, $\leq 0,35$, were discarded because they are not statistically significant.

Table 8 - Factor Matrix loadings (Varimax rotation method) for major and trace elements analysed from Site 212 core. Loadings less than 0,45 omitted (see above)

\begin{tabular}{|c|c|c|c|c|c|c|}
\hline & Factor 1 & Factor 2 & Factor 3 & Factor 4 & Factor 5 & Factor 6 \\
\hline Si & \begin{tabular}{|l|}
$-0,60$ \\
\end{tabular} & & 0,61 & $\mathbf{0 , 3 6}$ & & \\
\hline $\mathbf{T i}$ & & & 0,49 & 0,36 & 0,53 & \\
\hline Al & & & 0,91 & & & \\
\hline $\mathrm{Fe}_{\text {icotal) }}$ & 0,76 & & & & $-0,35$ & \\
\hline Mg & & & $-0,90$ & & & \\
\hline $\mathbf{C a}$ & & & & $-0,96$ & & \\
\hline $\mathbf{N a}$ & & $-0,88$ & & & & \\
\hline $\mathbf{K}$ & & 0,37 & $-0,37$ & & 0,74 & \\
\hline $\mathbf{P}$ & 0,74 & & 0,50 & & & \\
\hline $\mathrm{CO}_{2}$ & & & & $-0,95$ & & \\
\hline Mn & 0,97 & & & & & \\
\hline $\mathbf{v}$ & 0,76 & & 0,35 & & $-0,38$ & \\
\hline Cr & & & & & $-0,81$ & \\
\hline $\mathrm{Ni}$ & 0,93 & & & & & \\
\hline $\mathbf{C u}$ & 0,87 & & & & & \\
\hline $\mathrm{Zn}$ & & & 0,63 & & & $-0,43$ \\
\hline As & 0,77 & & 0,49 & & & \\
\hline $\mathbf{R b}$ & & 0,79 & & & 0,37 & \\
\hline Sr & 0,73 & & & & & \\
\hline $\mathbf{Y}$ & 0,88 & & & & & \\
\hline $\mathbf{Z r}$ & 0,66 & 0,36 & & 0,36 & & 0,38 \\
\hline Nh & 0,56 & & & & & 0,66 \\
\hline Mo & 0,91 & & & & & \\
\hline $\mathbf{B a}$ & & & & & 0,91 & \\
\hline La & & & 0,54 & 0,53 & & \\
\hline Ce & & & 0,66 & & & 0,41 \\
\hline $\mathbf{F b}$ & $\mathbf{0 , 9 0}$ & & & & & \\
\hline $\begin{array}{l}\text { Depth below } \\
\text { sea floor(in } \\
\text { m.) }\end{array}$ & $-0,83$ & & & & 0,33 & \\
\hline $\begin{array}{c}\% \text { of total } \\
\text { variance }\end{array}$ & 41,18 & 16,42 & 13,48 & 8,39 & 7,51 & 3,79 \\
\hline $\begin{array}{c}\text { Cumulative } \\
\%\end{array}$ & 41,18 & 57,59 & 71,08 & 79,47 & 86,97 & 90,76 \\
\hline
\end{tabular}

Table 9 - Correlations between varimax oblique factors for Site 212 values $\geq 0,30$ are shown since they are statistically more significant

\begin{tabular}{|c|c|c|c|c|c|c|}
\hline & F1 & F2 & F3 & F4 & F5 & F6 \\
\hline F1 & 1,00 & & 0,31 & & & 0,63 \\
F2 & & 1,00 & 0,35 & & & \\
F3 & 0,31 & 0,35 & 1,00 & & & 0,33 \\
F4 & & & & 1,00 & & \\
F5 & & & & & 1,00 & $-0,36$ \\
F6 & 0,63 & & 0,33 & & $-0,36$ & 1,00 \\
\hline
\end{tabular}

Factor 1 is a major one explaining the $41,17 \%$ of the total variance. This factor has high positive loadings for $\mathrm{Fe}, \mathrm{P}, \mathrm{Mn}, \mathrm{V}, \mathrm{Ni}, \mathrm{Cu}, \mathrm{As}, \mathrm{Sr}, \mathrm{Y}, \mathrm{Zr}, \mathrm{Mo}, \mathrm{Pb}$, medium positive values for $\mathrm{Nb}$ and strong negative values for $\mathrm{Si}$ and Depth. The positive loadings for the above elements represent the well known covariant group consisting the Fe-Mn hydroxides. The ability of manganese hydroxides to scavenge other transition metals such as $\mathrm{Cu}, \mathrm{Ni}, \mathrm{Pb}$ as well $\mathrm{Mo}$ is well known (Cronan 1969, Chester and Aston 1976, Chester 1990). The correlation of $\mathrm{P}$ with these oxides is not a surprise. According to Calvert et al. (1970), $\mathrm{P}_{2} \mathrm{O}_{5}$ usually concentrates in Fe-Mn 
micronodules. However, the high positive loadings of $\mathrm{Zr}$ and $\mathrm{Nb}$ indicate that this Fe-Mn oxide phase is associated at least partly with the detrital phase, mainly clay minerals. It is known that FeMn oxides coating clay minerals are able to adsorb large amounts of other elements from seawater. The medium positive correlation of this factor with Factor 3 representing the clay minerals and its strong positive correlation with Factor 6 (representing detrital minerals) (Table 9) confirms this conclusion. On the other hand the strong negative loading of depth in relation to positive loadings of Fe-Mn oxides phase indicate that these oxides are more abundant in the upper part of the borehole. This is shown also clearly in the $\mathrm{Fe} / \mathrm{Al}$ and $\mathrm{Mn} / \mathrm{Al}$ ratios versus depth in Figs 2 and 4. The strong negative correlation of Si represents mainly the biogenous opaline silica which is present in considerable amounts in some of the core sections analysed especially in the lower part of the borehole (Table 2). The strong negative loading of depth in this factor confirms this fact which is shown clearly in Fig. 2.

Factor 2 explains the $16,41 \%$ of the total variance and has positive loadings for Rb (high) and $\mathrm{K}$ (medium) and a high negative value for $\mathrm{Na}$. This factor will be discussed in combination with Factor 3 since there is medium positive correlation between those 2 factors.

Factor 3 explains the $13,48 \%$ of the total variance having strong to medium positive loadings for $\mathrm{Al}, \mathrm{Ti}, \mathrm{P}, \mathrm{Zn}, \mathrm{As}, \mathrm{La}, \mathrm{Ce}$ and strong to medium negative loadings for $\mathrm{Si}, \mathrm{Mg}, \mathrm{K}$. This factor represents the antipathetically related detrital clay minerals with authigenic minerals mainly zeolites and palygorskite and opaline silica. The strong to medium positive loadings of $\mathrm{Al}$ and $\mathrm{Ti}$ indicate that this factor represents detrital clay minerals which also mainly control the abundance and variance of, $\mathrm{Zn}, \mathrm{La}, \mathrm{Ce}$ and part of $\mathrm{P}$ and $\mathrm{As}$. The positive correlation of this factor with $\mathrm{F} 2$ means that the positive loadings of $\mathrm{F} 2$ i.e part of $\mathrm{K}$ and $\mathrm{Rb}$ are associated with the positive loadings of F3 i.e the detrital clay minerals. On the other hand the negative loadings of F2 i.e. Na are correlated with the negative loadings of $\mathrm{F} 3$ i.e $\mathrm{Si}, \mathrm{Mg}$ and partly $\mathrm{K}$ indicating that these elements represent the authigenic phases of opaline silica, palygorskite and zeolites mainly clinoptilolite.

Factor 4 explains the $8,39 \%$ of the total variance and represents the biogenic $\mathrm{CaCO}_{3}$ factor having very high negative loadings for $\mathrm{Ca}$ and $\mathrm{CO}_{2}$, contrasting the medium positive loadings for $\mathrm{Si}$, Ti, $\mathrm{Zr}$, La representing detrital silicate minerals mainly feldspars.

Factor 5 explains the $7,5 \%$ of the total variance having high to medium positive loadings for $\mathrm{Ti}$, $\mathrm{K}, \mathrm{Rb}$, and very high for $\mathrm{Ba}$, representing the sand fraction with a heavy mineral association (ilmenite, zircon, monazite) and barites. The very strong negative loadings of $\mathrm{Cr}$ and medium negative loadings of $\mathrm{Fe}_{\text {(total) }}$ and $\mathrm{V}$ most probably represent a $\mathrm{Fe}$ oxide phase. The medium negative correlation of this factor with factor 6 means that the negative loadings of F5 (i.e Cr, part of $\mathrm{Fe}_{\text {(total) }}$ and $\mathrm{V}$ ) are associated with the positive loadings of $\mathrm{F} 6$ (i.e $\mathrm{Zr}, \mathrm{Nb}, \mathrm{Ce}$ ) representing coatings of Fe hydroxides in detrital clays.

Factor 6 explains just the $3,79 \%$ of the total variance and has strong to medium positive loadings for $\mathrm{Nb}, \mathrm{Zr}$ and $\mathrm{Ce}$ and one medium negative loading for $\mathrm{Zn}$. The strong positive correlation of this factor with F1 (Table 9) implies that their positive loadings are associated confirming the fact that elements such as $\mathrm{Nb}, \mathrm{Zr}$, and $\mathrm{Ce}$ are associated with the main $\mathrm{Mn}-\mathrm{Fe}$ oxide phase represented by $\mathrm{F} 1$. The existence of the strong $\mathrm{Zr}$ loadings mean that these oxides are partly associated with detrital clays as coatings. This is confirmed from the medium positive correlation of F6 with F3 which implies that their positive loadings are related i.e part of $\mathrm{Zr}, \mathrm{Nb}$ and $\mathrm{Ce}$ are related with the positive loadings of F3 which represent the detrital clay minerals.

\subsubsection{Site 213}

After the principal components analysis, 5 factors, accounted for 91,54 of the total variance were extracted. The factor loadings for these 5 factors are shown in Table 10. Low factor loadings, $\leq 0,45$, were discarded because they are not statistically significant. 
In Table 11 the Varimax factor correlation matrix is shown aiding their interpretation.

Factor 1 explaining the $34,46 \%$ of the total variance has very high positive loadings for $\mathrm{Ti}, \mathrm{Fe}, \mathrm{P}$, $\mathrm{V}, \mathrm{Zn}, \mathrm{Ba}, \mathrm{La}, \mathrm{Ce}$ and high to medium positive loadings for $\mathrm{K}, \mathrm{Mn}, \mathrm{Ni}$ and $\mathrm{Pb}$. It represents the coexistence of Fe-Mn oxides with K-Feldspars and Plagioclase and other detrital minerals. This factor is strongly positively correlated with Factor 4 (Table 11) implying that the strong positive loadings ( $\mathrm{Mn}, \mathrm{Ni}, \mathrm{Cu}, \mathrm{Mo}, \mathrm{Pb}$ ) of $\mathrm{F} 4$ are associated with the loadings of F1 i.e. the abundant FeMn oxides phase in most of the core sections of Site 213. On the other hand the medium negative correlation of F1 with Factor 5 means that the strong to medium negative loadings of this factor (i.e $\mathrm{Mg}, \mathrm{Cr}$ ) are associated with this oxides phase implying that these oxides are associated with deep sea clays, very often coating their surface. It implies also a volcanogenic-hydrothermal origin associated with the basaltic basement of this Site. The fact that in Factor 1 there is also a very strong positive loading of depth confirms the fact that the abundance of $\mathrm{Fe}-\mathrm{Mn}$ oxides increases in the deeper part of Site 213. This is shown clearly in Fig. 3 where the $\mathrm{Mn} / \mathrm{Al}$ and $\mathrm{Fe} / \mathrm{Al}$ ratios are plotted versus depth.

Table 10 - Factor Matrix loadings (Varimax rotation method) for major and trace elements analysed from Site 213 cores. Loadings less than 0,45 omitted (see above)

\begin{tabular}{|c|c|c|c|c|c|}
\hline & Factor 1 & Facter 2 & Factor 3 & Factor 4 & Factor 5 \\
\hline Si & & 0,97 & & & \\
\hline $\mathbf{T i}$ & 0,90 & & & & \\
\hline Al & & 0,95 & & & \\
\hline Fe(total) & 0,91 & & & & \\
\hline $\mathbf{M g}$ & & 0,48 & & & $-0,58$ \\
\hline $\mathbf{C a}$ & & $-0,97$ & & & \\
\hline $\mathbf{N a}$ & & 0,69 & & & \\
\hline $\mathbf{K}$ & 0,66 & & & & 0,61 \\
\hline $\mathbf{P}$ & 0,86 & & & & \\
\hline $\mathrm{CO}_{2}$ & & $-0,97$ & & & \\
\hline Mn & 0,51 & & & $\mathbf{0 , 8 0}$ & \\
\hline $\mathbf{v}$ & 0,88 & & & & \\
\hline $\mathrm{Cr}$ & & $-0,52$ & & & $-0,51$ \\
\hline Ni & 0,46 & & & 0,76 & \\
\hline $\mathbf{C u}$ & & & & 0,85 & \\
\hline Zn & 0,92 & & & & \\
\hline As & & $-0,77$ & & & \\
\hline $\mathbf{F b}$ & & & 0,91 & & \\
\hline Sxr & & $-0,80$ & 0,53 & & \\
\hline $\mathbf{Y}$ & & & 0,82 & & \\
\hline $\mathbf{Z r}$ & & & 0,95 & & \\
\hline Nb & & & 0,79 & & \\
\hline Mo & & & 0,66 & 0,69 & \\
\hline $\mathbf{B a}$ & 0,68 & & & & \\
\hline La & 0,95 & & & & \\
\hline Ce & 0,91 & & & & \\
\hline $\mathbf{P b}$ & 0,65 & & & 0,71 & \\
\hline $\begin{array}{l}\text { Depth } \\
\text { below sea } \\
\text { floor }\end{array}$ & 0,71 & $-0,60$ & & & \\
\hline $\begin{array}{l}\text { Oo of total } \\
\text { variance }\end{array}$ & 34,47 & 26,99 & 16,18 & 9,25 & 4,48 \\
\hline $\begin{array}{l}\text { Cumulative } \\
\% \%\end{array}$ & 34,47 & 61,46 & 77,64 & 86,89 & 91,37 \\
\hline
\end{tabular}

Table 11 - Correlations between varimax oblique factors. Values $\geq 0,30$ are shown since are more statistically significant

\begin{tabular}{|c|c|c|c|c|c|}
\hline & F1 & F2 & F3 & F4 & F5 \\
\cline { 2 - 6 } F1 & 1,00 & & & 0,44 & $-0,38$ \\
F2 & & 1,00 & & & $-0,47$ \\
F3 & & & 1,00 & & \\
F4 & 0,44 & & & 1,00 & \\
F5 & $-0,38$ & $-0,47$ & & & 1,00 \\
\hline
\end{tabular}

Factor 2 explaining the $26,99 \%$ of the total variance has high positive loadings for $\mathrm{Si}, \mathrm{Al}, \mathrm{Na}$, a high to medium positive loading for $\mathrm{Mg}$ and high negative loadings for $\mathrm{Ca}, \mathrm{CO}_{2}, \mathrm{Cr}, \mathrm{As}, \mathrm{Sr}$. It 
represents the clay minerals and quartz $(\mathrm{Si}, \mathrm{Al}, \mathrm{Na}, \mathrm{Mg})$ antipathetically related with calcite $(\mathrm{Ca}$, $\mathrm{CO}_{2}, \mathrm{Sr}$ ). Calcite is mostly of biogenic origin in the present study. Chester (1990) stated that $\mathrm{Sr}$ is of biogenic origin and is supplied by the calcareous marine organisms. The association of $\mathrm{Sr}$ with biogenic carbonate has also been reported by Papavasiliou (1979), Turekian (1964), and Bostrom et. al. (1974). The negative loadings of $\mathrm{Cr}$ and $\mathrm{As}$ in this factor need however more explanation. $\mathrm{Cr}$ correlation with biogenic calcite could suggest the occurrence of $\mathrm{Cr}$ under reducing conditions (The organic tissue of calcareous fossils could provide the necessary organic matter for pyrite formation.). It is well known that under reducing conditions, $\mathrm{Cr}$ released from the alteration of basaltic material forms insoluble complexes and could exist as insoluble hydroxide or sulphide in the anoxic layer. In anoxic sediments As is associated often with organic material. In Site 213 samples the organic carbon content is substantially high with values up to 1,24\% (Table 3). So, in marine sediments association of $\mathrm{Cr}$ and As with organic carbon under reducing conditions is expected. The strong negative correlation of this factor with factor 5 (Table 11) implies that the strong to medium negative loadings of F5 $(\mathrm{Mg}, \mathrm{Cr})$ are associated with the positive loadings of F2 i.e the clay minerals association.

Factor 3 explaining the $16,18 \%$ of the total variance has high positive loadings for $\mathrm{Rb}, \mathrm{Y}, \mathrm{Zr}, \mathrm{Nb}$ and high to medium positive loadings for Mo and Sr. It represents detrital fraction of the sediments being rich in heavy minerals (i.e zircon,monazite) and feldspars whilst the presence of $\mathrm{Mo}$ and $\mathrm{Sr}$ in the positive loadings implies that the deposition of these detrital sediments took place in an anoxic environment. The diagenetic fixation of Mo to organic matter in a reducing environment is well established. (Krauskopf 1967, Calvert 1976, Hirst 1974).

Factor 4 explaining the $9,25 \%$ of the total variance has high positive loadings for $\mathrm{Mn}, \mathrm{Ni}, \mathrm{Cu}, \mathrm{Mo}$ and $\mathrm{Pb}$. All these elements form a well known covariant group. The ability of manganese hydroxides to scavenge other transition metals such as $\mathrm{Cu}, \mathrm{Ni} \mathrm{Zn}, \mathrm{Pb}$ as well $\mathrm{Mo}$ is well known (Cronan 1969, Chester and Aston 1976, Chester 1990). Thus Factor 4 represents a manganese hydroxide phase. This factor confirms the existence of "Mn oxide rich zeolitic clay" described in Table I where the main sedimentological characteristics of the presently studied cores where presented. The strong positive correlation of this factor with $\mathrm{F} 1$ confirms its association with $\mathrm{Fe}-$ oxides group as it has been mentioned commenting the Factor 1.

Factor 5 explaining the $4,48 \%$ of the total variance has high to medium negative loadings for $\mathrm{Mg}$, and $\mathrm{Cr}$ and a medium positive loading for $\mathrm{Cr}$ and a medium to negative loading for $\mathrm{K}$. Partly the positive loading of $\mathrm{Mg}$ represents the $\mathrm{Mg}$ rich authigenic mineral palygorskite which was found in some core sections in Site 213 (see mineralogy section). On the other hand the negative loading of $\mathrm{K}$ may represent the existence of the other authigenic mineral being present in considerable amounts in Site 213 core sections. This is the K-rich zeolite phillipsite (Table 3) which varies between 1-27\% in Site 213 core sections. On the other hand the high negative correlation of this factor with Factor 1 and mainly with Factor 2 (Table 11) implies that considerable amount of $\mathrm{Mg}$ and $\mathrm{Cr}$ are strongly associated with clay minerals and the detrital phases as it has been discussed earlier.

\section{Conclusions}

The cross examination of the clay mineralogy with the bulk chemical data including the examination of some of the metals to $\mathrm{Al}$ atomic ratio and the statistical factor analysis approach leads to a general as well as a few specific conclusions concerning Sites 212 and 213.

\section{A general conclusion}

The existence of the volcanogenic and authigenic (diagenetic or hydrothermal) inputs as well as the existence of the biogenous component in both 212 and 213 Sites has been confirmed later by similar findings in the Broken Ridge (south of Sites 212 and 213) in the border area of eastern Indian Ocean during the Ocean Drilling Project at ODP Site 752A (Owen and Zimmerman 1991). 


\subsection{Site 212}

1. The chemical composition of Site 212 sediments approaches more or less the composition of the typical deep sea clays. The sediments of this Site are composed mainly of expandable layered smectite/ illite having both authigenic and detrital origin, with considerable amounts of authigenic palygorskite and the zeolite clinoptilolite.

2. Fe-Mn oxides being more abundant in the upper part of Site 212 control to a large extent the abundance of $\mathrm{Fe}, \mathrm{Mn}, \mathrm{P}_{2} \mathrm{O}_{5}$ and trace elements like $\mathrm{V}, \mathrm{Ni}, \mathrm{Cu}, \mathrm{As}, \mathrm{Sr}, \mathrm{Mo}, \mathrm{Pb}, \mathrm{Ce}$. These oxides have been formed via diagenetic or hydrogenous processes coating very often the surface of deep sea clay particles. The strong association of these oxides with elements like $\mathrm{Zr}$ and $\mathrm{Nb}$ implies that part of these oxides are of detrital origin.

3. $\mathrm{Ca}$ is controlled mainly by biogenous $\mathrm{CaCO}_{3}$ whilst large amount of $\mathrm{SiO}_{2}$ is controlled by biogenous silica being present in several Site 212 core sections in considerable amount reaching values up to $18,15 \%$.

4. Authigenic clay mineral palygorskite and the zeolite clinoptilolite control to a large extent the abundance of $\mathrm{Mg}$ and part of $\mathrm{K}$ respectively.

5. The detrital heavy minerals and K-feldspars are controlling to a large extent the abundance of $\mathrm{Ti}, \mathrm{K}, \mathrm{Cr}, \mathrm{Zr}, \mathrm{Nb}$.

6. The increase of the $\mathrm{Ti} / \mathrm{Al}$ and $\mathrm{Mg} / \mathrm{Al}$ atomic ratios in the bottom samples of Site 212 implies a volcanogenic input in these sediments probably from the underlying basaltic basement. The increase in abundance of authigenic minerals like expandable layered smectite/illite and palygorskite, originated often by alteration of the basaltic material, supports further this conclusion.

\subsection{Site 213}

1. The average chemical composition of Site 213 sediments approaches also more or less the typical composition of deep sea clays. The Site 213 sediments are composed mainly of expandable layered smectite/illite having both authigenic and detrital origin, with large amounts of Fe-Mn oxides and considerable amounts of the authigenic zeolite phillipsite (up to $27 \%$ ) and the clay mineral palygorskite in small amounts only in the lower part of the borehole.

2. Large amount of Fe-Mn oxides being more abundant in the lower part of Site 213 control to a large extent the abundance of $\mathrm{Fe}, \mathrm{Mn}, \mathrm{P}_{2} \mathrm{O}_{5}$ and trace elements like $\mathrm{V}, \mathrm{Ni}, \mathrm{Cu}, \mathrm{Zn}, \mathrm{As}, \mathrm{Ba}, \mathrm{Mo}, \mathrm{Pb}$, $\mathrm{La}, \mathrm{Ce}$. The association of these oxides with $\mathrm{Ti}$ and part of $\mathrm{K}$ represents their coexistence with $\mathrm{K}$ Feldspars. However the considerable increase in abundance of Fe-Mn oxides in the lower part of the borehole, being in parallel with substantial increase of the $\mathrm{Ti} / \mathrm{Al}$ and $\mathrm{Mg} / \mathrm{Al}$ atomic ratios (Table 7 and Fig. 3) implies a strong hydrothermal and volcanogenic input in the formation of these oxides. The basaltic basement underneath Site 213 sediments further supports this conclusion.

3. The clay minerals are controlling the abundance of $\mathrm{Si}, \mathrm{Al}, \mathrm{Mg}$, and the largest part of $\mathrm{Na}$ and a proportion of $\mathrm{Cr}$, whilst biogenous $\mathrm{CaCO}_{3}$ is controlling the abundance of $\mathrm{Ca}, \mathrm{CO}_{2}$, and $\mathrm{Sr}$. The association of part of $\mathrm{Cr}$ and $\mathrm{As}$ with the biogenous fraction (i.e. calcite) especially in the lower part of the borehole is in accordance with the geochemical nature of these elements to form complex insoluble compounds under reducing conditions which seem to prevail in the lower part of Site 213. On the other hand the existence of the authigenic zeolite phillipsite controls considerable proportion of $\mathrm{K}$ and part of $\mathrm{Na}$.

4. Feldspars and the heavy detrital minerals are mainly controlling the abundance of elements like $\mathrm{Rb}, \mathrm{Zr}, \mathrm{Y}, \mathrm{Nb}$ and a small proportion of $\mathrm{Mo}$ and $\mathrm{Sr}$. 


\section{Acknowledgments}

The author gratefully acknowledges: (i) the Deep Sea Drilling Project and the U.S. National Science Foundation for their assistance and for the supply of sample material, (ii) the School of Ocean and Earth Science of the University of Southampton and the Institute de Mineralogie of the University of Leige for providing all the analytical facilities and carrying out the chemical analyses and clay mineralogy respectively.

\section{References}

Bostrom, K., Joensuu, O., and Brohm, I., 1974. Plankton-its chemical composition and its significance as a source of pelagic sediments, Chem.Geol., 14, 255-271.

Bostrom, K, Joensuu, O, Valdes, S, Charm, W, and Glaccum, R., 1976. Geochemistry and origin of East Pacific sediments sampled during DSDP leg 34, Initial Reports of the Deep Sea Drilling Project, Volume XXXIV, Washington (U .S. Government Printing Office), 559574pp.

Calvert, S.E., and Price, N.B., 1970. Minor metal contents of recent organic-rich sediments off South West Africa, Nature, 227, 593-595.

Calvert, S.E., 1976. The mineralogy and geochemistry of near-shore sediments. In J.P. Riley and R. Chester (eds). Chemical Oceanography, London Academic Press, 6, 187-280.

Chester, R., 1965. Elemental geochemistry of marine sediments. In J.P. Riley and G. Shirrow (eds), Chemical Oceanography, London Academic Press, 2, 23-80.

Chester, R., and Aston, R.S., 1976. The geochemistry of Deep Sea Sediments. In J.P. Riley and R. Chester (eds). Chemical Oceanography, London Academic Press, vol. 6, 281-390pp.

Chester, R., 1990. Marine Geochemistry, Published by Unwin Hyman Ltd. London , 700pp.

Cosgrove, M.E., and Papavasiliou, C.T., 1979. Clinoptilolite in DSDP sediments of the Indian Ocean (Site 223, Leg 23): Its stability conditions and estimation of its free energy, Marine.Geol., 19, 77-84.

Couture, R.A. 1977. Composition and origin of palygorskite-rich and montmorillonite- rich zeolite-containing sediments from the Pacific Ocean, Chem.Geol., 19, 113-130.

Cronan, D.S., 1969. Inter-element association in some pelagic deposits, Chem. Geol., 5, 99-106.

Donnely, T.W., and Wallace, J.L., 1976. Major and minor element chemistry of Antarctic clayrich sediments: Sites 322, 323, and 325, DSDP Leg.35. In Initial Reports of Deep Sea Drilling Project, vol. 35, Washington (U.S.Government Printing Office), 427-446pp.

Goldshmidt, V.M., 1954. Geochemistry, Oxford University Press, London, 730pp.

Griffin, J., Windom, H., and Goldberg, E., 1968. The distribution of clay minerals in the World Ocean, Deep Sea Reshearch, 15, 433-459.

Gromet, L.P., Dymek, R.F., Haskin, L.A., and Korotev, R.L., 1984. The "North American shale composite": its compilation, major and trace element characteristics, Geochim. Cosmochim. Acta, 48, 2469- 2482.

Hart, R.A., 1976. Chemical variance in deep ocean basalts, Initial Reports of Deep Sea Drilling Project, vol. 34, Washington (U.S.Government Printing Office), 301-339pp.

Hirst, M.D., 1974. Geochemistry of sediments from eleven Black Sea cores. In E.T. Degens and D.A. Ross (eds), The Black Sea geology, chemistry and Biology, Amer. Assoc.Petrol.Geol. Mem, 20, 430-455pp. 
Kolla, V., 1974.Mineralogical data from Sites 211, 212, 213, 214, 215 of Deep Sea Drilling Project Leg 22, and origin of non-carbonate sediments in the equatorial Indian Ocean, Initial Reports of Deep Sea Drilling Project, vol. 22, Washington (U.S.Government Printing Office), 489-501.

Kolla, V., Henderson, L., and Biscaye, P.E., 1976. Clay mineralogy and sedimentation in the Western Indian Ocean, Deep Sea Research, 23, 949-961.

Kolla,V., Kostecki, J.A., and Robinson, F., 1981. Sediments of region of clay minerals and quartz in surface sediments of the Arabian Sea, Journ.Sedim.Petrol., 51, 563-569.

Krauskopf, K.B., 1967. Introduction to Geochemistry,McGraw-Hill, New York, N.Y. 721pp.

Matti, J.C., Zemmels, I., and Cook, H.E., 1974. X-ray mineralogy data north-eastern part of the Indian Ocean, Leg 22, Deep Sea Drilling Project. In Initial Reports of Deep Sea Drilling Project, vol. 22, Washington (U.S.Government Printing Office), 693-710pp.

Owen, R.M., and Zimmerman, A.R.B., 1991. Geochemistry of Broken Ridge Sediments. In Proceedings of the Ocean Drilling Program, Scientific Results, vol.121, 437-445pp.

Papavasiliou, C.T., 1979. The geochemistry and mineralogy of some N.W. Indian Ocean cores (Sites 222 and 223, LEG.23, D.S.D.P., Unbubl. PhD thesis, University of Soutrhampton.

Pimm, A.C., 1974. Sedimentology and history of the north-eastern Indian Ocean from late Cretaceous to Recent. In Initial Reports of Deep Sea Drilling Project, vol. 22, Washington (U.S.Government Printing Office) 717-730pp.

Sclater, J.G., von der Borch, C.C., and Veevers, J.J., 1974. Regional synthesis of the deep sea drilling results from Leg. 22 in the eastern Indian Ocean. In Initial Reports of Deep Sea Drilling Project, vol. 22, Washington (U.S.Government Printing Office).

Subbaro, K.V., Kempe, D.R.C., Reddy, G.R., and Hekinian, 1979. Review of the geochemistry of Indian and other oceanic rocks. In Ahrens (ed.), Origin and Distribution of Elements: Second Symposium, Physics and Chemistry of the Earth, vol. II, Pergamon Press, Oxford, 367-399pp.

Taylor, S.R, and McLennan, S.M., 1985. The continental crust: its composition and evolution. An examination of the geochemical record preserved in sedimentary rocks, Geoscience Texts, Blackwell Scientific Publications, Oxford, 9-56pp.

Turekian, K.K., and Wedepohl, K.H., 1961. Distribution of the elements in some major units of the earth's crust, Bull.Geol.Soc.Amer. ,72, 175-192.

Turekian, K.K., 1964. The marine geochemistry of Strontium, Geochim. Cosmochim. Acta, 28 1479-1496.

Van der Weijden. C, Reichart, G.J , and Van Os, B., 2006. Sedimentary trace element records over the last $200 \mathrm{kyr}$ from within and below the northern Arabian Sea oxygen minimum zone, Marine Geology, 231, 69-88

Von der Borch, Sclater, J.G., et a1., 1974. Site Reports. In Initial Reports of Deep Sea Drilling Project, vol. 22, Washington (U.S.Govelment Printing Office).

Weser, O.E., 1974. Sedimentological aspects of strata encountered on Leg.23 in the northern Arabian Sea. In Initial Reports of Deep Sea Drilling Project, vol. 23, Washington (U.S. Government Printing Office), 503-519pp. 